\title{
Thermal Buckling Analysis of Sandwich Plates with Soft Core and CNT-Reinforced Composite Face Sheets
}

\author{
Saeed Kamarian ${ }^{\text {a }}$, Mahdi Bodaghi ${ }^{\mathrm{b}}$, Reza Barbaz Isfahani ${ }^{\mathrm{c}}$, Jung-il Song ${ }^{\text {à }}$ \\ a. Mechatronics Research Center, Changwon National University, Korea \\ b. Department of Engineering, School of Science and Technology, Nottingham Trent University, Nottingham, NG11 8NS, United Kingdom \\ c. Department of Mechanical Engineering, Amirkabir University of Technology, Tehran, Iran
}

\begin{abstract}
Previous studies on the thermal buckling of sandwich plates with composite face sheets indicate that only thin skins with high stiffness and low coefficients of thermal expansion (CTE) can lead to the desired buckling temperatures. Thus, carbon nanotubes (CNTs) that can significantly enhance thermo-mechanical properties of fibre-reinforced polymer composites are used in the present study to increase the critical buckling temperature of sandwich plates with soft core and laminated composite face sheets. First, a comprehensive series of experimental tests are conducted to evaluate the effects of nanotubes on thermomechanical properties of the face sheets. The experimental results indicate that using only $0.3 \% \mathrm{CNTs}$ considerably increases the longitudinal and transverse Young's modulus and shear modulus of the carbon fibre/epoxy composite face sheets. The obtained data also show that CNTs significantly decrease the CTE of composite skins. Subsequently, thermal buckling equations of sandwich plates with CNT-reinforced composite face sheets are derived based on piecewise low-order shear deformation theory (PLSDT). Three analytical, semi analytical, and numerical methods are used to investigate thermal buckling behaviour of the sandwich plates with various boundary conditions. To verify the results, several comparisons are performed, which show that the implemented methods can predict the buckling temperatures of sandwich plates with high accuracy. Finally, a parametric study is conducted to examine the effects of CNTs on the thermal buckling of sandwich plates for different length to thickness ratios, thicknesses of face sheets, stacking sequences of layers, and various types of boundary conditions. The results indicate that CNTs can increase the critical buckling temperature of sandwich plates by $22 \%-36 \%$, based on the layup, geometrical parameters, and boundary conditions.
\end{abstract}

Keywords: Sandwich plates; Thermal buckling; Carbon nanotubes; Experiments, Analytical solution, Numerical solution

\footnotetext{
${ }^{\dagger}$ Corresponding Author, E-mail: jisong@ @ changwon.ac.kr
} 


\section{Introduction}

The demand for sandwich structures is increasing on a daily bases in various industries, such as aerospace, automotive, and marine, due to their unique features. ${ }^{1,2}$ In many engineering applications, structures are exposed to high temperature, and thus their thermal buckling analysis is extremely important. The first studies in this area are probably the research performed by Watanabe et al. ${ }^{3,4}$ and $\mathrm{Ko}^{5}$ in the 1990s. Watanabe et al. ${ }^{3,4}$ analysed thermal buckling of sandwich plates with aluminium honeycomb core and carbon-reinforced fibre plastic face sheets using finite element (FE) method, and $\mathrm{Ko}^{5}$ studied buckling of sandwich plates with aluminium honeycomb core and titanium face sheets using minimum potential energy and FE approaches.

Based on higher-order shear deformation theory (HSDT), Babu and $\mathrm{Kant}^{6}$ applied two different FE formulations for thermal buckling investigation of rectangular sandwich plates with laminated face sheets. They presented a parametric study to illustrate the effects of various boundary conditions, thickness of layers, and angle of fibres on critical buckling temperature of the structures. The results indicated that the transverse normal strain and thermal expansion of the plates in the thickness direction can be ignored without the loss of precision. They also concluded that increasing the thickness of face sheets decreases the critical buckling temperature of sandwich plates. In another study, Kant and $\mathrm{Babu}^{7}$ investigated thermal buckling of skew sandwich plates with soft core and fibre-reinforced laminated face sheets. A comprehensive result was obtained for different boundary conditions, skew angle, thickness of layers, and lamination schemes. Shiau and $\mathrm{Kuo}^{8}$ performed a study to analyse thermal buckling of composite sandwich plates. They used a 36-degree of freedom plate element for the analysis by considering shear deformations of the core. The results showed that the fibre orientation can affect the critical buckling temperature and buckling modes. It was found that the optimum fibre orientation for a square sandwich plate with $( \pm \theta / \text { Core })_{s}$ laminated face sheets is $+45^{\circ}$ while this optimal value for a rectangular plate with an aspect ratio of more than 2 is approximately $+63^{\circ}$. The study also concluded that composite materials with high $E_{1} / E_{2}$ and low $\alpha_{1} / \alpha_{2}$ ratios exhibit high critical buckling temperature. Matsunaga ${ }^{9,10}$ examined thermal buckling of rectangular cross-ply and angle-ply composite and sandwich plates based on HSDT. In the studies, different truncated $m^{\text {th }}$-order theories were applied to obtain the critical buckling temperatures of the structures. The accuracy of the results was evaluated by comparing them with the data from threedimensional layer wise solution. It was observed that the implemented theories can predict the buckling temperature of sandwich plates with high accuracy. Vangipuram and Ganesan ${ }^{11}$ investigated buckling and free vibrations of sandwich plates under thermal effects. They performed a parametric study to demonstrate the effect of some parameters, such as core thickness, stacking sequence of layers, and boundary conditions 
on the behaviour of sandwich plates in buckling. The results indicated that the coupling thermal conductivity term did not significantly affect the critical buckling temperature of the structure. It was also observed that the optimal fibre orientation varies based on the type of boundary conditions. A decoupled thermo-mechanical analysis was presented by Pradeep and Ganesan ${ }^{12}$ to investigate thermal buckling and free vibration of multi-layered viscoelastic sandwich plates. The boundary conditions at all edges were considered as clamped. The researchers revealed variations in the critical buckling temperatures, natural frequencies, and mode shapes with respect to various effective parameters including core thickness. Han et al. ${ }^{13}$ developed a new, accurate, and simple refined theory to examine buckling and free vibration of foamfilled corrugated rectangular sandwich plates with simply supported boundary conditions by considering the thermal effect of environment. They showed variations in critical buckling temperature and natural frequencies relative to layup orientations, number of layers, and thickness of face sheets. The study suggested that foam filling decreases buckling temperature and fundamental frequencies of the structures by up to $13.7 \%$. Based on the sublaminate generalised unified formulation, Vescovini et al. ${ }^{14}$ analysed thermal buckling characteristics of laminated sandwich plates. The efficiency of the implemented approach was evaluated via comparing the results with those reported by three-dimensional solution. The buckling temperatures and modes were comprehensively examined for different material properties, geometrical parameters, and layup schemes. Active control of buckling and vibrations of simply supported sandwich plates with piezoelectric fibre-reinforced composite actuator face sheets under thermal environments was performed by $\mathrm{Li}$ et al. ${ }^{15}$ The analysis was based on classical plate theory (CPT) with von Karman nonlinearity terms. The results indicated that the use of a piezoelectric actuator significantly increases the critical buckling temperature and fundamental natural frequency of sandwich plates. It was also concluded that it is possible to maintain the fundamental natural frequency of the structure at a constant value under any operating temperature by implementing an appropriate stable gain control voltage. Zhai et al. ${ }^{16}$ focused on buckling temperature and natural frequencies of sandwich plates with soft core and single-layer face sheets. They presented an analytical solution for buckling and vibration of sandwich plates with simply supported type of boundary conditions under thermal environments and illustrated the influence of geometrical parameters on the behaviour of structure in free vibration and buckling.

CNTs are advanced materials with unique characteristics. Hence, over the last decade, they have attracted the attention of many researchers in the field of structural analysis and are widely used in engineering structures. Several studies have investigated the effect of CNTs on mechanical, thermal, and electrical properties of polymers. They showed that only a small additions of these materials (typically less than 0.5 wt $\%$ ) can considerably improve composite properties. ${ }^{17-20}$ Siddiqui et al. ${ }^{21}$ indicated that $0.3 \mathrm{wt} \%$ CNTepoxy nanocomposite coating significantly increases the tensile strength of a single fibre for all gage 
lengths, and this is higher than that due to neat epoxy coating. Barai and Weng ${ }^{22}$ experimentally and numerically revealed that CNT agglomeration and imperfect interface condition significantly decrease the effective stiffness and elasto-plastic strength. Thermo-mechanical effect of incorporating uniformly dispersed multi-walled carbon nanotubes (MWCNTs) into pristine and polyol-toughened epoxy was studied by Rahman et al. ${ }^{23}$ They showed that the addition of $0.3 \mathrm{wt} \%$ MWCNTs increases composite stiffness and decreases CTE such that it is lower than the CTE of epoxy-based resins. Recently, in a study ${ }^{24}$, experimental tests to demonstrate the effect of CNTs on the CTE of epoxy resin for different CNT loading were conducted. It was concluded that that increases in CNTs in polymer phase do not necessarily decrease the CTE of the polymer composite. Additionally, $0.3 \mathrm{wt} \% \mathrm{CNT}$ was reported as the optimum amount for nanotubes to yield the minimum CTE for epoxy resin.

A review of extant studies reveals a paucity of research on the effect of CNTs on thermal buckling of sandwich structures. Almost all studies considered functionally graded distribution for nanomaterials in the thickness direction of polymeric structures. Nonlinear buckling behaviour of sandwich spherical and conical shells with graded CNT-reinforced faces sheets was examined by Sankar et al. ${ }^{25}$. They performed a detailed parametric study to indicate the effect of the geometrical parameters and distribution and volume fraction of nanotubes on thermal buckling of the structure. Thermo-mechanical buckling of sandwich beams was conducted by Ebrahimi and Farazmandnia. ${ }^{26}$ They assumed that the beam is composed of stiff core and polymeric face sheets reinforced by functionally graded CNTs. The numerical results clarified that CNTs significantly increase critical buckling temperature of the structure. Mehar et al. ${ }^{27}$ performed a study to analyse buckling behaviour of functionally graded CNT-reinforced polymer sandwich shells under thermal loads based on HSDT. A parametric study was presented to demonstrate the effect of CNT distribution in the thickness direction, different boundary conditions, length-to-thickness ratio, and curvature on the behaviour of thermal buckling characteristic of the structures. Van Tung and Long ${ }^{28}$ focused on thermal buckling and post buckling of composite sandwich cylindrical panels. Two types of sandwich structures were examined, namely a sandwich panel with graded CNT-reinforced face sheets and homogenous core and a sandwich panel with graded CNT-reinforced and homogeneous face sheets. The analysis was conducted based on FSDT and by using Galerkin approach for simply supported boundary conditions. The effects of CNTs on the thermal and post buckling of the structure were investigated with respect to different parameters including CNT distribution and thickness of layers.

The main goal of the present study is to investigate the effect of CNTs on the thermal buckling of softcore sandwich plates with layered composite face sheets. To the best of the author's knowledge, extant studies do not address the role of CNTs in buckling behaviour of sandwich plates with laminated face sheets 
exposed to thermal environments. As previously mentioned, in all existing studies, ${ }^{25-28}$ thermal buckling of nanocomposite sandwich beams, shells, and panels were investigated by assuming that the structures consist of only pure polymers reinforced by graded nanotubes wherein their thermo-mechanical properties were estimated by an extended rule of mixture model with some limitations. However, in common engineering applications, composite structures usually include long and continuous fibres, such as carbon fibres or glass fibres in the form of laminates with different layups, and this requires more engineering coefficients in longitudinal and transverse directions of the fibres for their analysis. In the present study, CNTs are used as reinforcing phase for the epoxy matrix in the face sheets of sandwich plates along with long carbon fibres. All mechanical properties (i.e., longitudinal Young's modulus, transverse Young's modulus, shear modulus, and Poisson's ratio) are obtained by conducting some experiments. Additionally, the CTE of the hybrid composites is measured using a two-step procedure with the aid of both experiments and theoretical models. Therefore, more accurate results for thermal buckling analysis of the structure are obtained via experimental values of the material properties. Furthermore, governing equations and related boundary conditions relations for the thermal buckling problem are derived based on PLSDT, and solved for various types of boundary conditions. For simply supported sandwich plates, an analytical solution is provided that leads to a close-form solution for critical buckling temperatures. It is extremely complicated to determine an analytical solution for sandwich plates containing clamped boundary conditions. Therefore, in the case with two parallel simply supported and two clamped boundaries, a semi-analytical method is implemented such that a trigonometric solution is considered for the simply supported boundaries while generalised differential quadrature (GDQ) technique is applied to the clamped edges. This method exhibits simple formulation, low computational cost, and high accuracy when it is employed for the global behaviour analysis of structural elements such as bending, free vibration, and buckling problems. Furthermore, the strong form of the governing equations and the related boundary conditions are discretised in the method; hence, the shear locking phenomenon that occurs in conventional FE methods is absent. ${ }^{29,30}$ Finally, for fully clamped sandwich plates, two-dimensional GDQ approach is used for the analysis. A parametric study is then performed to illustrate the influences of CNTs on the thermal buckling of sandwich plates for different effective parameters such as length to thickness ratios, thicknesses of face sheets, layups, and boundary conditions.

\section{Problem description}

\subsection{Materials \& Experiments}

\subsubsection{Pure Materials}


Commercially available Araldite LY-5052 epoxy and Aradur HY-5052 hardener, purchased from Huntsman Corporation, were used as the main matrix component. The epoxy exhibits a low viscosity that makes dispersion of additives and fabrication of composite specimens easier at room temperature. MWCNTs were obtained from the US Research nanomaterials. Their characteristics included the following: purity > 95\%, outer diameters: 20-30 $\mathrm{nm}$, inner diameter 5-10 $\mathrm{nm}$, tube length range: 10-30 $\mathrm{mm}$, specific surface area $>110 \mathrm{~m}^{2} / \mathrm{g}$, and density: $2100 \mathrm{~kg} / \mathrm{m}^{3}$. High-performance unidirectional T-300 carbon fibre was used in the study.

\subsubsection{Preparation of the hybrid composite (carbon fibre/ CNT / epoxy)}

An optimal nanocomposite was prepared with $0.3 \mathrm{wt} \%$ CNTs, as reported in a previous study. ${ }^{24}$ First, the CNTs were mixed in Araldite LY-5052 epoxy resin via a per-mill mixer for $10 \mathrm{~min}$ at $500 \mathrm{rpm}$ and this was followed by ultrasonic mixing for $50 \mathrm{~min}$. During the sonication process, the container was kept in iced water to retain the nanocomposite temperature around room temperature. It was also stirred every $5 \mathrm{~min}$ using a small spoon to ensure that the sonication energy was homogeneously transferred to the entire mixture. The mixture was then placed in a vacuum oven at room temperature for $15 \mathrm{~min}$ to remove bubbles. After this stage, Aradur HY-5052 hardener was added to the CNT-reinforced epoxy and mixed at a low speed. As suggested by the manufacturer, Araldite LY-5052 epoxy resin and Aradur HY-5052 hardener were mixed at 100:38.2 weight ratio. Thin walled hybrid composite sheets were then fabricated using the hand layup approach as per ASTM standards for the required tests. Finally, the specimens were cut as per ASTM standards using waterjet cutting because it offers numerous advantages for trimming composites.

\subsubsection{Characterization of the hybrid composite}

Tensile tests in the longitudinal direction (LD) and transverse direction (TD) were performed using the samples as per ASTM/D3039 standard at room temperature. All tensile tests were conducted using a universal testing machine (SANTAM STM-50). Major Poisson's ratio was also measured as the ratio between transverse and longitudinal strains at the beginning of loading. In-plane shear tests were then conducted on the samples prepared according to ASTM D3518 to measure shear modulus using digital image correlation (DIC) method. Finally, mean LD and TD thermal expansion coefficients of the hybrid composite were measured. To this end, first, the CTE of CNT-reinforced epoxy was experimentally obtained as per ASTM E831 standard via thermo-mechanical analysis (TMA-500, Polymer Laboratories). Subsequently, the longitudinal and transverse CTE of the hybrid composite were calculated by using the 
micromechanical model described by Hyer. ${ }^{31}$ Table 1 summarizes the effects of CNTs on the thermomechanical properties of the carbon fibre/epoxy composite. This table indicates that the addition of $0.3 \mathrm{wt} \%$ CNTs increases the LD Young's modulus by $17.6 \%$, TD Young's modulus by $14.9 \%$, and shear modulus by $17.1 \%$ while it decreases the longitudinal CTE by $50 \%$ and transverse CTE by $26.5 \%$. It is also seen that CNTs do not have a significant effect on the major Poisson's ratio.

\subsection{Thermal buckling equations}

We consider a rectangular sandwich plate of dimension $a \times b \times h$, as shown in Fig. 1. The thicknesses of each face sheet and core are $h_{f}$ and $2 h_{c}$, respectively. Although the cores of sandwich structures are usually non-composite or non-laminated, a general formulation is provided for sandwich plates consisting of laminated composite cores and face sheets. Based on PLSDT, ${ }^{32,33}$ the displacement components for the core and face sheets of the structure are expressed as follows:

\section{Transverse displacement $\quad w(x, y, z)=w(x, y)$,}

component at any point:

In-plane displacement components
in the top and bottom face sheets: $\left\{\begin{array}{l}u_{t}(x, y, z)=u_{0}(x, y) \mp \frac{2 h_{c}+h_{f}}{2} \psi_{1}-\left(z \mp \frac{2 h_{c}+h_{f}}{2}\right) \frac{\partial w}{\partial x} \\ v_{t}(x, y, z)=v_{0}(x, y) \mp \frac{2 h_{c}+h_{f}}{2} \psi_{2}-\left(z \mp \frac{2 h_{c}+h_{f}}{2}\right) \frac{\partial w}{\partial y}\end{array}\right.$

In plane displacement components
in the core: $\left\{\begin{array}{l}u_{c}(x, y, z)=u_{0}(x, y)-z\left(\frac{2 h_{c}+h_{f}}{2 h_{c}} \psi_{1}-\frac{h_{f}}{2 h_{c}} \frac{\partial w}{\partial x}\right) \\ v_{c}(x, y, z)=v_{0}(x, y)-z\left(\frac{2 h_{c}+h_{f}}{2 h_{c}} \psi_{2}-\frac{h_{f}}{2 h_{c}} \frac{\partial w}{\partial y}\right)\end{array}\right.$

where index $c$ denotes the core layer, and indices $t$ and $b$ denote the upper and lower face sheets, respectively. Additionally, $\psi_{1}$ and $\psi_{2}$ denote the rotation angles of the straight line that connects the midpoint of the face sheets in $x o z$ and yoz planes, respectively. The von Karman-type non-linear strains for the face sheets are as follows: 


$$
\begin{aligned}
& \varepsilon_{t x}=\frac{\partial u_{t}}{\partial x}+\frac{1}{2}\left(\frac{\partial w}{\partial x}\right)^{2}=\left(\frac{\partial u_{0}}{\partial x} \mp \frac{2 h_{c}+h_{f}}{2} \frac{\partial \psi_{1}}{\partial x}+\frac{1}{2}\left(\frac{\partial w}{\partial x}\right)^{2}\right)+\left(z \mp \frac{2 h_{c}+h_{f}}{2}\right)\left(-\frac{\partial^{2} w}{\partial x^{2}}\right)={\stackrel{(0)}{\varepsilon_{t x}}}_{b}+\left(z \mp \frac{2 h_{c}+h_{f}}{2}\right)^{(1)} \varepsilon_{b x} \\
& \varepsilon_{t y}=\frac{\partial u_{t}}{\partial y}+\frac{1}{2}\left(\frac{\partial w}{\partial y}\right)^{2}=\left(\frac{\partial v_{0}}{\partial y} \mp \frac{2 h_{c}+h_{f}}{2} \frac{\partial \psi_{2}}{\partial y}+\frac{1}{2}\left(\frac{\partial w}{\partial y}\right)^{2}\right)+\left(z \mp \frac{2 h_{c}+h_{f}}{2}\right)\left(-\frac{\partial^{2} w}{\partial y^{2}}\right)={\stackrel{(0)}{\varepsilon_{t, y}}}_{b}+\left(z \mp \frac{2 h_{c}+h_{f}}{2}\right){\stackrel{(1)}{\varepsilon_{t}, y}}_{b} \\
& \gamma_{t x y}=\frac{\partial u_{t}}{\partial y}+\frac{\partial v_{t}}{\partial x}=\left(\left(\frac{\partial u_{0}}{\partial y}+\frac{\partial v_{0}}{\partial x}\right) \mp\left(\frac{2 h_{c}+h_{f}}{2}\right)\left(\frac{\partial \psi_{1}}{\partial y}+\frac{\partial \psi_{2}}{\partial x}\right)\right)+\left(z \mp \frac{2 h_{c}+h_{f}}{2}\right)\left(-2 \frac{\partial^{2} w}{\partial x \partial y}\right)=\gamma_{t b y}^{(0)}+\left(z \mp \frac{2 h_{c}+h_{f}}{2}\right) \gamma_{t, x y}^{(1)}
\end{aligned}
$$

Similarly, strain components for the core are expressed as follows:

$$
\begin{aligned}
& \varepsilon_{c x}=\frac{\partial u_{c}}{\partial x}+\frac{1}{2}\left(\frac{\partial w}{\partial x}\right)^{2}=\left(\frac{\partial u_{0}}{\partial x}+\frac{1}{2}\left(\frac{\partial w}{\partial x}\right)^{2}\right)+z\left(-\frac{2 h_{c}+h_{f}}{2 h_{c}} \frac{\partial \psi_{1}}{\partial x}+\frac{h_{f}}{2 h_{c}} \frac{\partial^{2} w}{\partial x^{2}}\right)=\stackrel{(0)}{\varepsilon}_{c x}+z \stackrel{(1)}{\varepsilon}_{c x} \\
& \varepsilon_{c y}=\frac{\partial v_{c}}{\partial y}+\frac{1}{2}\left(\frac{\partial w}{\partial y}\right)^{2}=\left(\frac{\partial v_{0}}{\partial y}+\frac{1}{2}\left(\frac{\partial w}{\partial y}\right)^{2}\right)+z\left(-\frac{2 h_{c}+h_{f}}{2 h_{c}} \frac{\partial \psi_{2}}{\partial y}+\frac{h_{f}}{2 h_{c}} \frac{\partial^{2} w}{\partial y^{2}}\right)=\stackrel{(0)}{\varepsilon}_{c y}+z \stackrel{(1)}{\varepsilon}_{c y} \\
& \gamma_{c x y}=\frac{\partial u_{c}}{\partial y}+\frac{\partial v_{c}}{\partial x}=\left(\frac{\partial u_{0}}{\partial y}+\frac{\partial v_{0}}{\partial x}\right)+z\left(-\frac{2 h_{c}+h_{f}}{2 h_{c}}\left(\frac{\partial \psi_{1}}{\partial y}+\frac{\partial \psi_{2}}{\partial x}\right)+\frac{h_{f}}{2 h_{c}}\left(2 \frac{\partial^{2} w}{\partial x \partial y}\right)\right)=\gamma_{c x y}^{(0)}+z \gamma_{c x y}^{(1)} \\
& \gamma_{c x z}=\frac{\partial u_{c}}{\partial z}+\frac{\partial w}{\partial x}=\frac{2 h_{c}+h_{f}}{2 h_{c}}\left(\frac{\partial w}{\partial x}-\psi_{1}\right) \\
& \gamma_{c y z}=\frac{\partial v_{c}}{\partial z}+\frac{\partial w}{\partial y}=\frac{2 h_{c}+h_{f}}{2 h_{c}}\left(\frac{\partial w}{\partial y}-\psi_{2}\right)
\end{aligned}
$$

When the structure is exposed to thermal environments, the stress components in the $k^{\text {th }}$ layer of the face sheets or core are calculated as follows:

$$
\begin{aligned}
& \left\{\begin{array}{c}
\sigma_{t x} \\
b \\
\sigma_{t y} \\
b \\
\sigma_{t x y} \\
b
\end{array}\right\}^{k t} b=\left[\begin{array}{lll}
\bar{Q}_{11} & \bar{Q}_{12} & \bar{Q}_{16} \\
& \bar{Q}_{22} & \bar{Q}_{26} \\
S y m & & \bar{Q}_{66}
\end{array}\right]^{k t}\left(\left\{\begin{array}{c}
\varepsilon_{t x} \\
b \\
\varepsilon_{t y} \\
b \\
\varepsilon_{t x y} \\
b
\end{array}\right\}^{k t}-\Delta T\left\{\begin{array}{c}
\alpha_{t x} \\
b \\
\alpha_{t y} \\
b \\
2 \alpha_{t x y} \\
b
\end{array}\right\}^{k t} b\right. \\
& \left\{\begin{array}{l}
\sigma_{c x} \\
\sigma_{c y} \\
\sigma_{c x y}
\end{array}\right\}^{k c}=\left[\begin{array}{lll}
\overline{Q_{11}} & \bar{Q}_{12} & \bar{Q}_{16} \\
& \bar{Q}_{22} & \bar{Q}_{26} \\
S y m & & \bar{Q}_{66}
\end{array}\right]^{k c}\left(\left\{\begin{array}{l}
\varepsilon_{c x} \\
\varepsilon_{c y} \\
\varepsilon_{c x y}
\end{array}\right\}^{k c}-\Delta T\left\{\begin{array}{l}
\alpha_{c x} \\
\alpha_{c y} \\
2 \alpha_{c x y}
\end{array}\right\}\right) \\
& \left\{\begin{array}{l}
\tau_{c y z} \\
\tau_{c x z}
\end{array}\right\}^{k c}=\left[\begin{array}{ll}
\bar{Q}_{44} & \bar{Q}_{45} \\
\bar{Q}_{45} & \bar{Q}_{55}
\end{array}\right]\left\{\begin{array}{l}
\gamma_{y z} \\
\gamma_{x z}
\end{array}\right\}^{k c}
\end{aligned}
$$

where $\bar{Q}_{i j}$ denotes the transformed reduced stiffness coefficients detailed in Appendix $1, \Delta T$ is the temperature variation, and $\alpha_{x}, \alpha_{y}$ and $\alpha_{x y}$ are the in-plane thermal expansion coefficients defined in Appendix 2. Furthermore, $k t, k b$, and $k c$ denote the $k^{\text {th }}$ layer in top face, bottom face, and the core, respectively. It should be noted that some assumptions are considered in the present study as follows:

- Top and bottom face sheets are similar.

- Th layup of face sheets is cross-ply and symmetric with respect to their mid-planes. 
- Temperature distribution is uniform.

By using the aforementioned assumptions, the stress resultants in the face sheets are calculated as follows:

$$
\begin{aligned}
& \left\{\begin{array}{c}
N_{t x} \\
b \\
N_{t y} \\
b \\
N_{t x y} \\
b
\end{array}\right\}=\left[\begin{array}{ccc}
A_{f 11} & A_{f 12} & 0 \\
A_{f 12} & A_{f 22} & 0 \\
0 & 0 & A_{f 66}
\end{array}\right]\left\{\begin{array}{c}
(0) \\
\varepsilon_{t x} \\
b \\
(b) \\
\varepsilon_{b y} \\
b \\
(0) \\
\gamma_{t y y} \\
b y
\end{array}\right\}-\left\{\begin{array}{l}
N_{f x}^{T} \\
N_{f y}^{T} \\
0
\end{array}\right\} \\
& \left\{\begin{array}{c}
M_{t x} \\
b \\
M_{t y}^{t y} \\
M_{t x y} \\
b
\end{array}\right\}=\left[\begin{array}{ccc}
D_{f 11} & D_{f 12} & 0 \\
D_{f 12} & D_{f 22} & 0 \\
0 & 0 & D_{f 66}
\end{array}\right]\left\{\begin{array}{c}
(1) \\
\mathcal{E}_{t x} \\
b \\
(1) \\
\mathcal{E}_{b y} \\
b \\
\gamma_{b} \\
\gamma_{t y}
\end{array}\right\}
\end{aligned}
$$

where $A_{f i j}$ and $D_{f i j}$ are the extensional and bending stiffness coefficients for the face sheets, respectively, which are defined as follows:

$$
\left(A_{f j j}, D_{f j i}\right)=\int_{h_{c}}^{h_{c}+h_{f}} \bar{Q}_{i j_{\text {face }}}\left[1,\left(z-\frac{2 h_{c}+h_{f}}{2}\right)^{2}\right] d z=\int_{-h_{c}-h_{f}}^{-h_{c}} \bar{Q}_{i j_{f a c e}}\left[1,\left(z+\frac{2 h_{c}+h_{f}}{2}\right)^{2}\right] d z=\int_{-h_{f} / 2}^{h_{f} / 2} \bar{Q}_{i j_{\text {face }}}\left(1, z^{2}\right) d z
$$

In Eq. (5), $N_{f x}^{T}$ and $N_{f y}^{T}$ denote the in-plane thermal forces in the sheets that are calculated as follows:

$$
\left\{\begin{array}{l}
N_{f x}^{T} \\
N_{f y}^{T}
\end{array}\right\}=\left\{\begin{array}{l}
N_{t x}^{T} \\
N_{t y}^{T}
\end{array}\right\}=\left\{\begin{array}{l}
N_{b x}^{T} \\
N_{b y}^{T}
\end{array}\right\}=\int_{-h_{f} / 2}^{h_{f} / 2}\left[\begin{array}{ll}
\bar{Q}_{11} & \bar{Q}_{12} \\
\bar{Q}_{12} & \bar{Q}_{22}
\end{array}\right]_{\text {face }}\left\{\begin{array}{l}
\alpha_{x} \\
\alpha_{y}
\end{array}\right\}_{\text {face }} \Delta T d z
$$

Similarly, the stress resultants for the core are also obtained as follows:

$$
\begin{aligned}
& \left\{\begin{array}{l}
N_{c x} \\
N_{c y} \\
N_{c x y}
\end{array}\right\}=\left[\begin{array}{ccc}
A_{c 11} & A_{c 12} & 0 \\
A_{c 12} & A_{c 22} & 0 \\
0 & 0 & A_{c 66}
\end{array}\right]\left\{\begin{array}{l}
(0) \\
\mathcal{E}_{c x} \\
(0) \\
\mathcal{E}_{c y} \\
(0) \\
\gamma_{c x y}
\end{array}\right\}-\left\{\begin{array}{l}
N_{c x}^{T} \\
N_{c y}^{T} \\
0
\end{array}\right\} \\
& \left\{\begin{array}{l}
M_{c x} \\
M_{c y} \\
M_{c x y}
\end{array}\right\}=\left[\begin{array}{ccc}
D_{c 11} & D_{c 12} & 0 \\
D_{c 12} & D_{c 22} & 0 \\
0 & 0 & D_{c 66}
\end{array}\right]\left\{\begin{array}{l}
(1) \\
\mathcal{E}_{c x} \\
(1) \\
\mathcal{E}_{c y} \\
(1) \\
\gamma_{c x y}
\end{array}\right\}
\end{aligned}
$$

in which,

$$
\begin{aligned}
& \left(A_{c i j}, D_{c i j}\right)=\int_{-h_{c}}^{h_{c}} \bar{Q}_{i j_{c o r e}}\left(1, z^{2}\right) d z \\
& \left\{\begin{array}{l}
N_{c x}^{T} \\
N_{c y}^{T}
\end{array}\right\}=\int_{-h_{c}}^{h_{c}}\left[\begin{array}{ll}
\bar{Q}_{11} & \bar{Q}_{12} \\
\bar{Q}_{12} & \bar{Q}_{22}
\end{array}\right]_{c o r e}\left\{\begin{array}{l}
\alpha_{x} \\
\alpha_{y}
\end{array}\right\}_{c o r e} \Delta T d z
\end{aligned}
$$

In the next step, the strain energy of the sandwich plate is calculated as follows: 


$$
\begin{aligned}
& U=U_{t}+U_{b}+U_{c} \\
& U_{t}=U_{t x}+U_{t y}+U_{t x y}=\frac{1}{2} \int_{0}^{a} \int_{0}^{b} \int_{h_{c}}^{h_{c}+h_{f}} \sigma_{t x} \varepsilon_{t x} d z d y d x+\frac{1}{2} \int_{0}^{a} \int_{0}^{b} \int_{h_{c}}^{h_{c}+h_{f}} \sigma_{t y} \varepsilon_{t y} d z d y d x+\int_{0}^{a} \int_{0}^{b} \int_{h_{c}}^{h_{c}+h_{f}} \tau_{t x y} \gamma_{t x y} d z d y d x \\
& U_{b}=U_{b x}+U_{b y}+U_{b x y}=\frac{1}{2} \int_{0}^{a} \int_{0}^{b} \int_{-h_{c}-h_{f}}^{-h_{c}} \sigma_{b x} \varepsilon_{b x} d z d y d x+\frac{1}{2} \int_{0}^{a} \int_{0}^{b} \int_{-h_{c}-h_{f}}^{-h_{c}} \sigma_{b y} \varepsilon_{b y} d z d y d x+\int_{0}^{a} \int_{0}^{b} \int_{-h_{c}-h_{f}}^{-h_{c}} \tau_{b x y} \gamma_{b x y} d z d y d x \\
& U_{c}=U_{c x}+U_{c y}+U_{c x y}+U_{c x z}+U_{c y z}=\frac{1}{2} \int_{0}^{a} \int_{0}^{b} \int_{-h_{c}}^{h_{c}} \sigma_{c x} \varepsilon_{c x} d z d y d x+\frac{1}{2} \int_{0}^{b} \int_{0}^{h_{c}} \int_{-h_{c}} \sigma_{c y} \varepsilon_{c y} d z d y d x \\
& +\int_{0}^{a} \int_{0}^{b} \int_{-h_{c}}^{h_{c}} \tau_{c x y} \gamma_{c x y} d z d y d x+\int_{0}^{a} \int_{0}^{b} \int_{-h_{c}}^{h_{c}} \tau_{c x z} \gamma_{c x z} d z d y d x+\int_{0}^{a} \int_{0}^{b} \int_{-h_{c}}^{h_{c}} \tau_{c y z} \gamma_{c y z} d z d y d x
\end{aligned}
$$

Based on the principle of virtual work, ${ }^{34}$ in the absence of external loads, we have the following expression:

$$
\delta U=0
$$

By inserting Eq. (10) into Eq. (11), five governing equations are obtained in which two are related to inplane equations and the remaining three equations are related to out-of-plane equations. By implementing pre-buckling analysis for the sandwich plate with immovable simply supported and clamped boundary conditions, thermal buckling equations of the structure are expressed as follows:

$$
\begin{aligned}
& g_{11}^{(3)} \frac{\partial^{4} w}{\partial x^{4}}+\left(2 g_{12}^{(3)}+4 g_{66}^{(3)}\right) \frac{\partial^{4} w}{\partial x^{2} \partial y^{2}}+g_{22}^{(3)} \frac{\partial^{4} w}{\partial y^{4}}+g_{11}^{(2)} \frac{\partial^{3} \psi_{1}}{\partial x^{3}}+\left(g_{12}^{(2)}+2 g_{66}^{(2)}\right)\left(\frac{\partial^{3} \psi_{1}}{\partial x \partial y^{2}}+\frac{\partial^{3} \psi_{2}}{\partial x^{2} \partial y}\right)+ \\
& g_{22}^{(2)} \frac{\partial^{3} \psi_{2}}{\partial y^{3}}+S_{c x z}\left(\frac{\partial \psi_{1}}{\partial x}-\frac{\partial^{2} w}{\partial x^{2}}\right)+S_{c y z}\left(\frac{\partial \psi_{2}}{\partial y}-\frac{\partial^{2} w}{\partial y^{2}}\right)+N_{x}^{T} \frac{\partial^{2} w}{\partial x^{2}}+N_{y}^{T} \frac{\partial^{2} w}{\partial y^{2}}=0 \\
& -g_{11}^{(2)} \frac{\partial^{3} w}{\partial x^{3}}-\left(g_{12}^{(2)}+2 g_{66}^{(2)}\right) \frac{\partial^{3} w}{\partial x \partial y^{2}}-\left(g_{12}^{(1)}+g_{66}^{(1)}\right) \frac{\partial^{2} \psi_{2}}{\partial x \partial y}-g_{11}^{(1)} \frac{\partial^{2} \psi_{1}}{\partial x^{2}}-g_{66}^{(1)} \frac{\partial^{2} \psi_{1}}{\partial y^{2}}+S_{c x z}\left(\psi_{1}-\frac{\partial w}{\partial x}\right)=0 \\
& -g_{22}^{(2)} \frac{\partial^{3} w}{\partial y^{3}}-\left(g_{12}^{(2)}+2 g_{66}^{(2)}\right) \frac{\partial^{3} w}{\partial x^{2} \partial y}-\left(g_{12}^{(1)}+g_{66}^{(1)}\right) \frac{\partial^{2} \psi_{1}}{\partial x \partial y}-g_{22}^{(1)} \frac{\partial^{2} \psi_{2}}{\partial y^{2}}-g_{66}^{(1)} \frac{\partial^{2} \psi_{2}}{\partial x^{2}}+S_{c y z}\left(\psi_{2}-\frac{\partial w}{\partial y}\right)=0
\end{aligned}
$$

where the coefficients of $g_{i j}^{(k)}$ are presented in Appendix 3, and $N_{x}^{T}$ and $N_{y}^{T}$ are obtained as follows:

$$
\left(\begin{array}{c}
N_{x}^{T} \\
N_{y}^{T}
\end{array}\right)=\left(\begin{array}{c}
N_{c x}^{T} \\
N_{c y}^{T}
\end{array}\right)+\left(\begin{array}{c}
N_{t x}^{T} \\
N_{t y}^{T}
\end{array}\right)+\left(\begin{array}{c}
N_{b x}^{T} \\
N_{b y}^{T}
\end{array}\right)=\left(\begin{array}{c}
N_{c x}^{T} \\
N_{c y}^{T}
\end{array}\right)+2\left(\begin{array}{c}
N_{f x}^{T} \\
N_{f y}^{T}
\end{array}\right)
$$

The related boundary conditions are also derived as follows:

$$
\begin{array}{ll}
x=0, a: \begin{cases}\delta w=0 & \text { or }\left(-g_{11}^{(2)} \frac{\partial^{2} \psi_{1}}{\partial x^{2}}-g_{11}^{(3)} \frac{\partial^{3} w}{\partial x^{3}}-g_{12}^{(2)} \frac{\partial^{2} \psi_{2}}{\partial x \partial y}-g_{12}^{(3)} \frac{\partial^{3} w}{\partial x \partial y^{2}}+S_{c x z}\left(\frac{\partial w}{\partial x}-\psi_{1}\right)\right)=0 \\
\delta\left(\frac{\partial w}{\partial x}\right)=0 & \text { or }\left(g_{11}^{(2)} \frac{\partial \psi_{1}}{\partial x}+g_{11}^{(3)} \frac{\partial^{2} w}{\partial x^{2}}+g_{12}^{(2)} \frac{\partial \psi_{2}}{\partial y}+g_{12}^{(3)} \frac{\partial^{2} w}{\partial y^{2}}\right)=0 \\
\delta \psi_{1}=0 & \text { or }\left(g_{11}^{(1)} \frac{\partial \psi_{1}}{\partial x}+g_{11}^{(2)} \frac{\partial^{2} w}{\partial x^{2}}+g_{12}^{(1)} \frac{\partial \psi_{2}}{\partial y}+g_{12}^{(2)} \frac{\partial^{2} w}{\partial y^{2}}\right)=0\end{cases} \\
y=0, b: \begin{cases}\delta w=0 & \text { or }\left(-g_{22}^{(2)} \frac{\partial^{2} \psi_{2}}{\partial y^{2}}-g_{22}^{(3)} \frac{\partial^{3} w}{\partial y^{3}}-g_{12}^{(2)} \frac{\partial^{2} \psi_{1}}{\partial x \partial y}-g_{12}^{(3)} \frac{\partial^{3} w}{\partial x^{2} \partial y}+S_{c y z}\left(\frac{\partial w}{\partial y}-\psi_{2}\right)\right)=0 \\
\delta\left(\frac{\partial w}{\partial y}\right)=0 & \text { or }\left(g_{22}^{(2)} \frac{\partial \psi_{2}}{\partial y}+g_{22}^{(3)} \frac{\partial^{2} w}{\partial y^{2}}+g_{12}^{(2)} \frac{\partial \psi_{1}}{\partial x}+g_{12}^{(3)} \frac{\partial^{2} w}{\partial x^{2}}\right)=0 \\
\delta \psi_{2}=0 & \text { or }\left(g_{22}^{(1)} \frac{\partial \psi_{2}}{\partial y}+g_{22}^{(2)} \frac{\partial^{2} w}{\partial y^{2}}+g_{12}^{(1)} \frac{\partial \psi_{1}}{\partial x}+g_{12}^{(2)} \frac{\partial^{2} w}{\partial x^{2}}\right)=0\end{cases}
\end{array}
$$


It is worth noting that for the clamped boundary conditions, we have $\delta w=\delta\left(\frac{\partial w}{\partial x}\right)=\delta\left(\frac{\partial w}{\partial y}\right)=\delta \psi_{1}=\delta \psi_{2}=0$, thus, Eqs. (14) are automatically satisfied.

\subsection{Solution methods}

This subsection attempts to explain as to how to solve thermal buckling equations of the rectangular sandwich plate with different boundary conditions, as shown in Eqs. (12) and (13). Three different methods are provided based on the type of boundaries.

Simply supported boundary conditions (SSSS)

For sandwich plates with simply supported boundary conditions at all the edges, an analytical solution is suggested as follows:

$$
\begin{aligned}
& w(x, y)=W_{m n} \sin \frac{m \pi}{a} x \sin \frac{n \pi}{b} y \\
& \psi_{1}(x, y)=\Psi_{1 m n} \cos \frac{m \pi}{a} x \sin \frac{n \pi}{b} y \\
& \psi_{2}(x, y)=\Psi_{2 m n} \sin \frac{m \pi}{a} x \cos \frac{n \pi}{b} y
\end{aligned}
$$

where $m$ and $n$ denote the number of half-wave in $x$ and $y$-directions. It is observed that the purposed solution can satisfy the boundary conditions in Eq. (14). Inserting Eq. (15) into Eq. (12) leads to the following expression:

$$
\left[\begin{array}{lll}
k_{11} & k_{12} & k_{13} \\
k_{21} & k_{22} & k_{23} \\
k_{31} & k_{32} & k_{33}
\end{array}\right]\left[\begin{array}{l}
W_{m n} \\
\Psi_{1 m n} \\
\Psi_{2 m n}
\end{array}\right]=\left[\begin{array}{l}
0 \\
0 \\
0
\end{array}\right]
$$

where the coefficients of $k_{i j}$ are expressed in Appendix 4. Subsequently, a nontrivial solution is obtained for the above equations as follows:

$$
\operatorname{det}\left[\begin{array}{lll}
k_{11} & k_{12} & k_{13} \\
k_{21} & k_{22} & k_{23} \\
k_{31} & k_{32} & k_{33}
\end{array}\right]=0
$$

Solving Eq. (17) straightforwardly yields the critical buckling temperature in the form of:

$$
\Delta T_{c r}=\frac{f_{1}-f_{2}}{f_{0}}
$$

where 


$$
\begin{aligned}
& f_{0}=\left(\frac{m \pi}{a}\right)^{2} \tilde{N}_{x}^{T}+\left(\frac{n \pi}{b}\right)^{2} \tilde{N}_{y}^{T} \\
& f_{1}=\left(\frac{m \pi}{a}\right)^{4} g_{11}^{(3)}+\left(\frac{m \pi}{a}\right)^{2}\left(\frac{n \pi}{b}\right)^{2}\left(2 g_{12}^{(3)}+4 g_{66}^{(3)}\right)+\left(\frac{n \pi}{b}\right)^{4} g_{22}^{(3)}+\left(\frac{m \pi}{a}\right)^{2} S_{c x z}+\left(\frac{n \pi}{b}\right)^{2} S_{c y z} \\
& f_{2}=\left(k_{12}\left(k_{13} k_{23}-k_{12} k_{33}\right)+k_{13}\left(k_{12} k_{23}-k_{13} k_{22}\right)\right) /\left(k_{23}^{2}-k_{22} k_{33}\right) \\
& \left(\begin{array}{c}
\tilde{N}_{x}^{T} \\
\tilde{N}_{y}^{T}
\end{array}\right)=\left(\begin{array}{l}
\tilde{N} c_{x}^{T} \\
\tilde{N} c_{y}^{T}
\end{array}\right)+2\left(\begin{array}{l}
\tilde{N} f_{x}^{T} \\
\tilde{N} f_{y}^{T}
\end{array}\right)=\int_{-h_{c}}^{+h_{c}}\left[\begin{array}{ll}
\bar{Q}_{11} & \bar{Q}_{12} \\
\bar{Q}_{12} & \bar{Q}_{22}
\end{array}\right]_{\text {core }}\left\{\begin{array}{l}
\alpha_{x} \\
\alpha_{y}
\end{array}\right\}_{\text {core }} d z+2 \int_{-h_{f} / 2}^{+h_{f} / 2}\left[\begin{array}{ll}
\bar{Q}_{11} & \bar{Q}_{12} \\
\bar{Q}_{12} & \bar{Q}_{22}
\end{array}\right]_{\text {face }}\left\{\begin{array}{l}
\alpha_{x} \\
\alpha_{y}
\end{array}\right\}_{\text {face }} d z
\end{aligned}
$$

From Eq. (18), buckling temperatures of the structure are calculated for different values of $m$ and $n$. The minimum obtained value is considered as the critical buckling temperature, as denoted by symbol $\Delta T_{c r}$.

\section{Two opposite edges simply supported and two clamped boundary conditions at other edges (CSCS)}

In this case, the boundary conditions are assumed as clamped at $x=0, a$ albeit simply supported at $y=0, b$. A semi analytical solution is presented for sandwich plates with this type of boundaries. It is observed that by adopting the following form for the displacement components, the boundary conditions at $y=0, b$ are satisfied.

$$
\begin{aligned}
& w(x, y)=W(x) \sin \frac{n \pi}{b} y \\
& \psi_{1}(x, y)=\Psi_{1}(x) \sin \frac{n \pi}{b} y \\
& \psi_{2}(x, y)=\Psi_{2}(x) \cos \frac{n \pi}{b} y
\end{aligned}
$$

Substituting Eq. (20) into Eq. (12) leads to the following equations:

$$
\begin{aligned}
& p_{1} \frac{d^{4} W}{d x^{4}}+p_{2} \frac{d^{2} W}{d x^{2}}+p_{3} W+p_{4} \frac{d^{3} \Psi_{1}}{d x^{3}}+p_{5} \frac{d \Psi_{1}}{d x}+p_{6} \frac{d^{2} \Psi_{2}}{d x^{2}}+p_{7} \Psi_{2}=0 \\
& p_{8} \frac{d^{3} W}{d x^{3}}+p_{9} \frac{d W}{d x}+p_{10} \frac{d^{2} \Psi_{1}}{d x^{2}}+p_{11} \Psi_{1}+p_{12} \frac{d \Psi_{2}}{d x}=0 \\
& p_{13} \frac{d^{2} W}{d x^{2}}+p_{14} W+p_{15} \frac{d \Psi_{1}}{d x}+p_{16} \frac{d^{2} \Psi_{2}}{d x^{2}}+p_{17} \Psi_{2}=0
\end{aligned}
$$

where the coefficients of $p_{i}$ are given in Appendix 5. It should be noted that coefficients $p_{2}$ and $p_{3}$ depend on temperature ( $\Delta T$ ). The GDQ method is employed to solve the aforementioned partial differential equations in the $x$ direction. Based on the technique, the $m^{\text {th }}$-order partial derivative of a function $f(x)$ with respect to $x$ at a given point $x_{i}$ is estimated as follows:

$$
\frac{\partial^{m} f}{\partial x^{m}}=\sum_{k=1}^{N_{x}} c_{i k}^{m} f\left(x_{k}\right) \quad ; \quad i=1,2, \ldots, N_{x} \quad, \quad m=2,3, \ldots N_{x}-1
$$

where $c_{i k}^{m}$ denotes the weighting coefficients which can be found in previous works. ${ }^{35,36}$ By applying GDQ approach into Eq. (21), the following discretised equations are obtained. 


$$
\begin{aligned}
& \sum_{k=1}^{N_{x}}\left(p_{1} c_{i k}^{4}+p_{2} c_{i k}^{2}\right) W\left(x_{k}\right)+p_{3} W\left(x_{i}\right)+\sum_{k=1}^{N_{x}}\left(p_{4} c_{i k}^{3}+p_{5} c_{i k}^{1}\right) \Psi_{1}\left(x_{k}\right)+\sum_{k=1}^{N_{x}} p_{6} c_{i k}^{2} \Psi_{2}\left(x_{k}\right)+p_{7} \Psi_{2}\left(x_{i}\right)=0 \\
& \sum_{k=1}^{N_{x}}\left(p_{8} c_{i k}^{3}+p_{9} c_{i k}^{1}\right) W\left(x_{k}\right)+\sum_{k=1}^{N_{x}} p_{10} c_{i k}^{2} \Psi_{1}\left(x_{k}\right)+p_{11} \Psi_{1}\left(x_{i}\right)+\sum_{k=1}^{N_{x}} p_{12} c_{i k}^{1} \Psi_{2}\left(x_{k}\right)=0 \\
& \sum_{k=1}^{N_{k}} p_{13} c_{i k}^{2} W\left(x_{k}\right)+p_{14} W\left(x_{i}\right)+\sum_{k=1}^{N_{x}} p_{15} c_{i k}^{1} \Psi_{1}\left(x_{k}\right)+\sum_{k=1}^{N_{k}} p_{16} c_{i k}^{2} \Psi_{2}\left(x_{k}\right)+p_{17} \Psi_{2}\left(x_{i}\right)=0
\end{aligned}
$$

After discretizing the buckling equations, the eigenvalue problem is obtained as follows:

$$
[\mathbf{S}]\left\{\begin{array}{l}
\left\{W_{d}\right\} \\
\left\{\Psi_{1 d}\right\} \\
\left\{\Psi_{2 d}\right\}
\end{array}\right\}=\{0\}
$$

in which $[\mathbf{S}]$ denotes the stiffness matrix and $\left\{W_{d}\right\},\left\{\Psi_{1 d}\right\}$, and $\left\{\Psi_{2 d}\right\}$ are defined as follows:

$$
\left\{W_{d}\right\}=\left\{\begin{array}{l}
W_{2} \\
W_{3} \\
\ldots \\
W_{N_{x}-1}
\end{array}\right\} \quad, \quad\left\{\psi_{1 d}\right\}=\left\{\begin{array}{l}
\Psi_{1_{2}} \\
\Psi_{1_{3}} \\
\ldots \\
\Psi_{1_{N_{x}-1}}
\end{array}\right\} \quad, \quad\left\{\Psi_{2 d}\right\}=\left\{\begin{array}{l}
\Psi_{2_{2}} \\
\Psi_{2_{3}} \\
\ldots \\
\Psi_{2_{N_{x}-1}}
\end{array}\right\}
$$

It should be noted that generally $[\mathbf{S}]=\left[S_{d d}\right]-\left[S_{d b}\right]\left[S_{b b}\right]^{-1}\left[S_{b d}\right]$, and this is obtained by discretizing governing equations (12) and boundary conditions (14). However, the boundaries at edges $x=0, a$ are clamped, and thus $\left[S_{b d}\right]$ becomes zero and $[\mathbf{S}]=\left[S_{d d}\right]$. Therefore, only the thermal buckling equations are required to be discretised. To obtain the critical buckling temperature of the structure, for different values of $n, \Delta T$ is gradually increased until the determinant of the matrix [S] becomes zero. The lowest value is considered as the critical temperature of the structure.

\section{Fully clamped boundary conditions (CCCC)}

Fully clamped sandwich plates are considered here as the third type of boundary conditions. To obtain the critical buckling temperature for this case, a two-dimensional GDQ approach is implemented to approximate the partial derivatives of a continuous function $f(x, y)$ at point $(x, y)=\left(x_{i}, y_{j}\right)$ as:

$$
\begin{aligned}
& \left.\frac{\partial^{m} f}{\partial x^{m}}\right|_{\left(x_{i}, y_{j}\right)}=\sum_{k=1}^{N_{k}} c_{i k}^{m} f\left(x_{k}, y_{j}\right) \\
& \left.\frac{\partial^{n} f}{\partial y^{n}}\right|_{\left(x_{i}, y_{j}\right)}=\sum_{l=1}^{N_{y}} \bar{c}_{j l}^{n} f\left(x_{i}, y_{l}\right) \\
& \left.\frac{\partial^{(m+n)} f}{\partial x^{m} \partial y^{n}}\right|_{\left(x_{i}, y_{j}\right)}=\sum_{k=1}^{N_{k}} \sum_{l=1}^{N_{y}} c_{i k}^{m} \bar{c}_{j l}^{n} f\left(x_{k}, y_{l}\right)
\end{aligned}
$$


where $c_{i k}^{m}$ and $\bar{c}_{j l}^{n}$ denote the weighting coefficients for $x$ and $y$ directions, respectively. By applying twodimensional GDQ method to Eq. (12), the following discretised equations are obtained.

$$
\begin{aligned}
& g_{11}^{(3)} \sum_{k=1}^{N_{*}} c_{i k}^{4} w\left(x_{k}, y_{j}\right)+\left(2 g_{12}^{(3)}+4 g_{66}^{(3)}\right) \sum_{k=1}^{N_{k}} \sum_{l=1}^{N_{y}} c_{i k}^{2} \bar{c}_{j l}^{2} w\left(x_{k}, y_{l}\right)+g_{22}^{(3)} \sum_{l=1}^{N_{y}} \bar{c}_{j l}^{4} w\left(x_{i}, y_{l}\right)+g_{11}^{(2)} \sum_{k=1}^{N_{x}} c_{i k}^{3} \psi_{1}\left(x_{k}, y_{j}\right)+ \\
& \left(g_{12}^{(2)}+2 g_{66}^{(2)}\right)\left(\sum_{k=1}^{N_{x}} \sum_{l=1}^{N_{y}} c_{i k}^{1} \bar{c}_{j l}^{2} \psi_{1}\left(x_{k}, y_{l}\right)+\sum_{k=1}^{N_{x}} \sum_{l=1}^{N_{y}} c_{i k}^{2} \bar{c}_{j l}^{1} \psi_{2}\left(x_{k}, y_{l}\right)\right)+g_{22}^{(2)} \sum_{l=1}^{N_{y}} \bar{c}_{j l}^{3} \psi_{2}\left(x_{i}, y_{l}\right)+ \\
& S_{c x z}\left(\sum_{k=1}^{N_{x}} c_{i k}^{1} \psi_{1}\left(x_{k}, y_{j}\right)-\sum_{k=1}^{N_{x}} c_{i k}^{2} w\left(x_{k}, y_{j}\right)\right)+S_{c y z}\left(\sum_{l=1}^{N_{y}} \bar{c}_{j l}^{1} \psi_{2}\left(x_{i}, y_{l}\right)-\sum_{l=1}^{N_{y}} \bar{c}_{j l}^{2} w\left(x_{i}, y_{l}\right)\right)+ \\
& N_{x}^{T} \sum_{k=1}^{N_{x}} c_{i k}^{2} w\left(x_{k}, y_{j}\right)+N_{y}^{T} \sum_{l=1}^{N_{y}} \bar{c}_{j l}^{2} w\left(x_{i}, y_{l}\right)=0 \\
& -g_{11}^{(2)} \sum_{k=1}^{N_{k}} c_{i k}^{3} w\left(x_{k}, y_{j}\right)-\left(g_{12}^{(2)}+2 g_{66}^{(2)}\right) \sum_{k=1}^{N_{k}} \sum_{l=1}^{N_{y}} c_{i k}^{1} \bar{c}_{j l}^{2} w\left(x_{k}, y_{l}\right)-\left(g_{12}^{(1)}+g_{66}^{(1)}\right) \sum_{k=1}^{N_{k}} \sum_{l=1}^{N_{y}} c_{i k}^{1} \bar{c}_{j l}^{1} \psi_{2}\left(x_{k}, y_{l}\right) \\
& -g_{11}^{(1)} \sum_{k=1}^{N_{k}} c_{i k}^{2} \psi_{1}\left(x_{k}, y_{j}\right)-g_{66}^{(1)} \sum_{l=1}^{N_{v}} \bar{c}_{j l}^{2} \psi_{2}\left(x_{i}, y_{l}\right)+S_{c x z}\left(\psi_{1}\left(x_{i}, y_{j}\right)-\sum_{k=1}^{N_{x}} c_{i k}^{1} w\left(x_{k}, y_{j}\right)\right)=0 \\
& -g_{22}^{(2)} \sum_{l=1}^{N_{v}} \bar{c}_{j l}^{3} w\left(x_{i}, y_{l}\right)-\left(g_{12}^{(2)}+2 g_{66}^{(2)}\right) \sum_{i=1}^{N_{x}} \sum_{k=1}^{N_{v}} c_{i k}^{2} \bar{c}_{j l}^{1} w\left(x_{k}, y_{l}\right)-\left(g_{12}^{(1)}+g_{66}^{(1)}\right) \sum_{i=1}^{N_{x}} \sum_{k=1}^{N_{v}} c_{i k}^{1} \bar{c}_{j l}^{1} \psi_{1}\left(x_{k}, y_{l}\right) \\
& -g_{22}^{(1)} \sum_{l=1}^{N_{y}} \bar{c}_{j l}^{2} \psi_{2}\left(x_{i}, y_{l}\right)-g_{66}^{(1)} \sum_{k=1}^{N_{k}} c_{i k}^{2} \psi_{2}\left(x_{k}, y_{j}\right)+S_{c y z}\left(\psi_{2}\left(x_{i}, y_{j}\right)-\sum_{l=1}^{N_{y}} \bar{c}_{j l}^{1} w\left(x_{i}, y_{l}\right)\right)=0
\end{aligned}
$$

The above equations can be also re-expressed in the following compact form.

$$
[\mathbf{S}]\left\{\begin{array}{l}
\left\{w_{d}\right\} \\
\left\{\psi_{1_{d}}\right\} \\
\left\{\psi_{2_{d}}\right\}
\end{array}\right\}=\{0\}
$$

where

$$
\begin{aligned}
& \left\{w_{d}\right\}=\left\{w_{2,2}, w_{2,3}, \ldots, w_{2, N_{y}-1}, \ldots, w_{N_{x}-1,2}, w_{N_{x}-1,3}, \ldots, w_{N_{x}-1, N_{y}-1}\right\}^{T} \\
& \left\{\psi_{1_{d}}\right\}=\left\{\psi_{1_{2,2}}, \psi_{1_{2,3}}, \ldots, \psi_{1_{2, N_{y}-1}}, \ldots, \psi_{1_{N_{x}-1,2}}, \psi_{1_{N_{x}-1,3}}, \ldots, \psi_{1_{N_{x}-1, N_{y}-1}}\right\}^{T} \\
& \left\{\psi_{2_{d}}\right\}=\left\{\psi_{2_{2,2}}, \psi_{2,3}, \ldots, \psi_{2_{2, N_{y}-1}}, \ldots, \psi_{2_{N_{x}-1,2}}, \psi_{2_{N_{x}-1,3}}, \ldots, \psi_{2_{N_{x}-1, N_{y}-1}}\right\}^{T}
\end{aligned}
$$

To obtain the critical buckling temperatures including that discussed for plates with CSCS boundary conditions, $\Delta T$ is gradually increased until the determinant of the matrix becomes zero. The temperature is reported as the critical temperature.

\section{Results and discussion}

\subsection{Convergence and verification study}

As previously mentioned, in the present study, thermal buckling of sandwich plates with CNT-reinforced laminated face sheets are analysed for various boundary conditions. For sandwich plates with simply 
supported boundaries, an analytical solution is provided while one-dimensional and two-dimensional GDQ techniques are employed for CSCS and CCCC sandwich plates, respectively. Specifically, a convergence study is first presented to demonstrate the performance of GDQ method for thermal buckling analysis. Thus, Table 2 shows the critical buckling temperatures of sandwich plates with nanocomposite face sheets for two different boundary conditions. Results for CSCS sandwich plates indicate that the use of only 7 grid points in the $x$-direction obtains converged buckling temperatures. As shown in Table 2, the responses converge by employing $9 \times 9$ grid points for fully clamped sandwich plates. Therefore, the GDQ method converges fast in both boundary conditions.

Subsequently, given the other goal of the subsection, a comparative study is presented to verify the analytical/semi analytical and numerical results. First, the present results are compared with those reported by $\mathrm{Ko}^{5}$ and Shiau and $\mathrm{Kuo}^{8}$ for thermal buckling of sandwich plates with aluminium honeycomb core and isotopic titanium face sheets given the following material properties:

$$
\begin{aligned}
& \text { Honeycomb Core }: E_{x}=E_{y}=2.7778 \times 10^{4} \mathrm{lb} / \mathrm{in}^{2} ; G_{x y}=0.00613 \times 10^{6} \mathrm{lb} / \mathrm{in}^{2} \\
& \qquad \begin{array}{c}
G_{x z}=1.81 \times 10^{5} \mathrm{lb} / \mathrm{in}^{2} ; G_{y z}=0.81967 \times 10^{5} \mathrm{lb} / \mathrm{in}^{2} \\
v_{x y}=0.658 \times 10^{-2} ; \alpha_{x}=\alpha_{y}=5.37 \times 10^{-6} \mathrm{in} / \mathrm{in}-{ }^{o} \mathrm{~F}
\end{array} \\
& \text { Face sheets }: E_{x}=E_{y}=13.1 \times 10^{6} \mathrm{lb} / \mathrm{in}^{2} ; G_{x y}=5.0 \times 10^{6} \mathrm{lb} / \mathrm{in}^{2} \\
& v_{x y}=0.31 ; \alpha_{x}=\alpha_{y}=5.35 \times 10^{-6} \mathrm{in} / \mathrm{in}-{ }^{o} \mathrm{~F}
\end{aligned}
$$

The comparison results are listed in Table 3. As shown in the table, the developed methods in the present study estimate the buckling temperature of sandwich plates with acceptable accuracy. Specifically, the present results are evidently extremely close to those obtained from the energy method reported by Ko, ${ }^{5}$ and also to the results obtained by using the 36-DOF triangular element reported by Shiau and Kuo ${ }^{8}$. The performance of the applied methods is also shown in Figs. 2 and 3 for the case $b / a=3$. It is observed that thermal buckling occurs at $(n, m)=(1,1)$ and $n=3$ for plates with SSSS and CSCS boundary conditions, respectively. The second comparison involves thermal buckling of a rectangular sandwich plate with soft core and single layer orthotropic face sheets $\left(0^{\circ} /\right.$ Core $\left./ 0^{\circ}\right)$. Zhai et al. ${ }^{16}$ used an analytical solution for this type of sandwich plate with only simply supported boundary conditions. The material properties of the core and face sheets are assumed as follows:

Isotropic Core $: E=10 \mathrm{MPa} ; v_{12}=0.3 ; \alpha=0.18 \times 10^{-6} /{ }^{\circ} \mathrm{C}$
Face sheets $: E_{1}=132 \mathrm{GPa} ; E_{2}=10.3 \mathrm{GPa} ; G_{12}=6.5 \mathrm{GPa} ;$
$v_{12}=0.3 ; \alpha_{1}=1.2 \times 10^{-6} /{ }^{\circ} \mathrm{C} ; \alpha_{2}=24 \times 10^{-6} /{ }^{\circ} \mathrm{C}$ 
It should be noted that the results by Zhai et al. ${ }^{16}$ are plotted as figure, and thus digitizer software with high accuracy is used to determine the numerical values of the critical buckling temperatures. The comparison shown in Fig. 4 indicates that the present results are in good agreement with those reported by Zhai et al. ${ }^{16}$ In the third example, the critical buckling temperature parameter of a non-sandwich four-layer $\left[0^{\circ} / 90^{\circ}\right]_{s}$ composite square plate with SSSS and CCCC boundary conditions are predicted and compared with the FE results obtained in the study by Kant and Babu. ${ }^{7}$ Thus, the thickness of the face sheets is set to zero $\left(h_{f}=0\right)$. The thickness to length ratio for the plate is assumed as 0.01 , and the material properties are as follows:

$$
\begin{aligned}
& \frac{E_{1}}{E_{2}}=15, \frac{G_{12}}{E_{2}}=0.5, \frac{G_{23}}{E_{2}}=0.3356, v_{12}=0.3, \\
& \frac{\alpha_{1}}{\alpha_{0}}=0.015, \frac{\alpha_{2}}{\alpha_{0}}=1, \alpha_{0}=10^{-6} /{ }^{\circ} \mathrm{C}
\end{aligned}
$$

The numerical results are tabulated in Table 4 and compared with their counterparts reported by Kant and Babu. $^{7}$ As shown in the table, the results for the simply supported case are evidently exactly identical. Additionally, for the clamped case, they are extremely close to each other and the difference between them is negligible.

Based on the aforementioned comparative studies, it is concluded that the employed analytical/semi analytical and numerical methods in the study exhibit sufficient accuracy to handle thermal buckling problem for sandwich plates with soft core and nanocomposite hybrid face sheets under various boundary conditions.

\subsection{Thermal buckling analysis}

A parametric study is performed to indicate the effect of CNTs on the thermal buckling of rectangular sandwich plates with different effective parameters. It should be noted that unless otherwise specified, in all other examples, the laminated composite face sheets are assumed as composed of three cross-ply layers of $\left[0^{\circ} / 90^{\circ} / 0^{\circ}\right]$. Furthermore, for the purpose of simplicity, $\Delta T_{c r}$ is termed as the critical buckling temperature.

Table 5 shows the effect of CNTs on the critical buckling temperatures of simply supported sandwich plates for different geometrical parameters. The first point that is inferred from the results of the table is that CNTs increase the buckling temperature of the structure for all lengths, widths, and face sheet thickness values. The enhancement varies from 23.2 to $29.5 \%$, based on the geometrical parameters. It is also 
observed that CNTs can change the critical values of $m$ and $n$. We consider a sandwich plate with $a=0.5 m, b=0.75 m, h_{f} / h=0.15$ as an example. The analytical results reveal that $(m, n)$ varies from $(1,1)$ to $(1$, 2) after using CNTs, and this is because the minimum buckling temperature obtained from Eq. 18 is a function of stiffness, geometrical parameters, and number of half-waves. When the stiffness of the structure changes because CNTs are added, the critical buckling temperature can occur at different $m$ and $n$. As shown in Table 5, for all length and width values, the critical buckling temperature of the structure decreases with increases in the parameter $h_{f} / h$ from 0.05 to 0.15 . It is also observed that the role of CNTs in delaying the buckling temperature of structure decreases with increases in the $h_{f} / h$ ratio. For example, in the case $a=0.5 \mathrm{~m}, b=0.5 \mathrm{~m}$, CNTs increase the critical temperature of the structure by approximately $28.5 \%$ if $h_{f} / h=0.05$ while the critical temperature of the structure increases by $26 \%$ when $h_{f} / h=0.15$. This is because increases in the thickness of the face sheets often increase the overall stiffness of the structures and decreases the positive role of CNTs.

For increased detail, Fig.5 shows the variations in the buckling temperature relative to the $h_{f} / h$ ratio for three different values of $(a, b)$. As shown in the figure, increases in the $h_{f} / h$ ratio from 0 to 0.3 initially decrease the critical temperature to a minimum amount and subsequently increase it. To justify the phenomenon, it is necessary to consider the effect of two main factors on the behaviour of structures in thermal environments, namely the stiffness of structure and the in-plane compressive thermal forces generated in the structure. Therefore, thickening face sheets exhibit two effects, i.e., a positive effect on stiffness coefficients $\left(g_{11}^{(1)}, g_{11}^{(2)}, \ldots\right)$ and a negative effect on $\tilde{N}_{x}^{T}$ and $\tilde{N}_{y}^{T}$. Thus, Fig. 6 shows the variations in $g_{11}^{(1)}, g_{11}^{(3)}$, and $\tilde{N}_{x}^{T}$ relative to the $h_{f} / h$ ratio. As shown in the figure, $\tilde{N}_{x}^{T}$ and the stiffness coefficients increase with the increase of $h_{f} / h$ parameter. Therefore, at a specific face sheet thickness, the effect of one can overwhelm the other. Thus, the aforementioned type of a decreasing-increasing trend is observed in Fig. 5. However, the CNTs evidently simultaneously increase the coefficient stiffness and decrease compressive in-plane thermal forces, thereby increasing critical buckling temperature at all face sheet thickness values based on Fig. 5. Figure 7 shows the increase rate of critical buckling temperature of sandwich plates due to the use of CNTs in composite face sheets for different $a, b$, and $h_{f} / h$ parameters. The results indicate that the buckling temperature of the sandwich plate can increase between approximately 21 to $31 \%$ based on the geometrical parameters. 
In the next step, the role of CNTs in thermal buckling behaviour of sandwich plates is examined from a design viewpoint. The thermal buckling behaviour of sandwich plates relative to $h_{f} / h$ ratio is discussed again as the first design point. As previously mentioned, the buckling temperature usually decreases with increases in the $h_{f} / h$ ratio. Thus, only very thin face sheets are mainly desirable for the enhancement of thermal buckling. Conversely, the total stiffness of a structure is extremely important for engineering applications, and thus engineers occasionally consider possible mechanical loads that can be applied on the structure. As shown in Fig. 6, thin face sheets do not lead to large or sufficient stiffness coefficients. Therefore, they do not satisfy the strength needed to cope with different situations. The role of CNTs is then investigated for the square sandwich plate discussed in Fig. 5 when $a=0.5 m, b=0.5 m$. It is assumed that $\Delta T_{c r}$ should exceed $50^{\circ} \mathrm{C}$ for a specific application. Thus, without using CNTs, $h_{f} / h$ should be less than 0.1 while CNTs can enhance this limit to 0.14 . Therefore, designers obtain a wider range of options for designing sandwich structure with composite face sheets.

In the second point of design, it should be noted that one of the important parameters in designing sandwich structures is to determine their core thickness. Also, while constructing these types of structures, the emphasis is always on minimizing the amount of required materials and thus the weight of the structure. Figure 8 shows the minimum required thickness of the core in terms of operating temperature to prevent buckling. As expected, increase in the operating temperature increase the core thickness of sandwich plates. The results plotted in the figure indicate that the use of CNTs decreases the minimum required core thickness for the design to avoid the buckling phenomenon. Evidently, the use of CNTs increases the stiffness of the upper and lower surfaces while simultaneously decreasing the compressive thermal forces generated in them. Thus, thinner cores are needed for designing. It is also observed from Fig. 8 that as the thickness of face sheets increases, CNTs play a more effective role in reducing the minimum required core thickness.

The effect of length to thickness parameter on the critical buckling temperature of a square sandwich plate with simply supported boundary conditions is examined. As shown in Fig. 9, decreases in the thickness of sandwich plate (increases in $a / h$ ) decrease the critical buckling temperature. Analytical results demonstrate that CNTs enhance thermal buckling of sandwich plates for all $a / h$ ratios. It is also observed that the diagrams of $h_{f} / h=0.1$ show lower critical temperatures when compared to $h_{f} / h=0.05$ and $h_{f} / h=0.2$, as shown in Fig. 5. Another figure is provided to illustrate the effect of CNTs on the buckling of square sandwich plates in the thermal environment with respect to $a / h$ parameter. As shown in Fig. 10, for 
sandwich plates without CNT in which $a / h$ ranges from 15 to 30 , the diagram for $h_{f} / h=0.1$ is completely above the diagrams for $h_{f} / h=0.15$. However, as previously mentioned, given some design limitations, additional thickness of face sheets is required, and this significantly decreases buckling temperature. In this condition, CNTs can play a significant positive role in behaviour of the structure. The results shown in Fig. 10 show that the use of CNTs increases the critical buckling temperature such that for $a / h \leq 21$, the buckling temperature for plate with thicker face sheets exceeds that of plate with thinner face sheets. Figure 11 shows the effect of using nanocomposite face sheets in a sandwich structure for thermal buckling enhancement. As shown in the figure, based on $a / h$ and $h_{f} / h$ ratios, critical buckling temperatures of the structure can increase up to approximately $36 \%$.

The role of nanotubes in buckling behaviour of sandwich plates is examined for different stacking sequences of layers of face sheets. Hence, four different layups $\left[0^{\circ} / 90^{\circ} / 0^{\circ}\right],\left[90^{\circ} / 0^{\circ} / 90^{\circ}\right],\left[0^{\circ} / 90^{\circ}\right]_{s}$, and $\left[90^{\circ} / 0^{\circ}\right]_{s}$ are considered for face sheets, and the related results are tabulated in Table 6. Additionally, the results represent that CNTs positively affect thermal buckling of sandwich plates with laminated face sheets for all layups. Furthermore, for the considered sandwich plate, CNTs can delay the critical buckling temperature of the structure by $22.3-25.8 \%$ based on the orientation of fibres. Moreover, the results of the table indicate that CNTs can change the critical half-wave numbers based on stacking sequence of layers.

Finally, the influence of using CNTs on buckling temperature of sandwich plates are investigated with respect to different types of boundary conditions. Tables 7 and 8 show that positive effect of nanotubes on the buckling temperature occur in all types of boundary conditions. However, the effect of CNTs on sandwich plates with simply supported boundaries exceeds that on clamped ones. This is because increases in clamp boundaries make the structure stiffer and reduce the positive role of CNTs on the thermal buckling behaviour. Variations in buckling temperature with respect to the aspect ratio are shown in Figs. 12 and 13 for sandwich plates with simply supported and fully clamped boundary conditions. A decrease-increase pattern is observed for variations in buckling temperature of simply supported condition. Conversely, for the fully clamped plate, critical buckling temperature of the structure almost always decrease with increases in the aspect ratio. However, the figures clearly indicate that the effect of CNTs increases with increases in the aspect ratio. The results in Fig. 14 illustrates that based on boundary conditions, CNTs increase the critical buckling temperature of the specified sandwich structure in the range of $22 \%$ to $29 \%$. 


\section{Conclusion}

In the present study, thermal buckling of rectangular sandwich plates with layered composite skins was examined. The core was assumed as composed of a soft material while a laminated hybrid composite consisted of CNTs, carbon fibre, and epoxy polymer as face sheets. All required mechanical properties of the nanocomposite sheets were obtained by conducting experimental tests. Thermal expansion coefficients of the composite were also obtained via a two-step process using experimental methods and micromechanical models. In the next step, governing equations for thermal buckling behaviour of sandwich plates with cross-ply laminated face sheets were derived based on PLSDT, and solved for different boundary conditions using three analytical, semi analytical, and numerical methods. The conclusions drawn from the study can be summarized as follows:

- CNTs omprove thermomechanical features of composite materials such that the mechanical properties including Young's modulus and shear modulus increase while CTE of the composite significantly decreases.

- Proper governing equations and related boundary condition relations for thermal buckling analysis of sandwich plates can be derived via PLSDT.

- CNTs increase the critical buckling temperature of sandwich plates for all boundary conditions, geometrical parameters, and layups.

- The results indicate that based on the boundary conditions and geometrical properties, the increase in the critical buckling temperature due to the use of CNTs ranges from approximately $22 \%$ to $36 \%$.

- The use of CNTs in composite face sheets decreases the thickness required for the core and thereby decreases the weight of the structure.

- CNTs allow the use of thicker composite face sheets for sandwich plates while preventing buckling phenomenon.

\section{Acknowledgement}

The study was supported by the National Research Foundation of Korea (NRF) grant funded by the Korea government (2018R1A6A1A03024509, 2019R1A2B5B03004980). 


\section{References}

[1] Birman, V. and Kardomateas, G.A., 2018. Review of current trends in research and applications of sandwich structures. Composites Part B: Engineering, 142, pp.221-240.

[2] Sayyad, Atteshamuddin S., and Yuwaraj M. Ghugal. "On the free vibration analysis of laminated composite and sandwich plates: A review of recent literature with some numerical results." Composite Structures 129 (2015): 177-201.

[3] Watanabe, Naoyuki, and Kensuke Teranishi. "Stiffness and buckling analysis for sandwich plates with thermal buckling CFRP sheets-Qualitative approach." In 35th Structures, Structural Dynamics, and Materials Conference, p. 1575. 1994.

[4] Watanabe, Naoyuki, Hiroyuki Sato, Yasushi Hishi, Naoyuki Watanabe, Hiroyuki Sato, and Yasushi Hishi. "Thermal buckling behavior in Al honeycomb sandwich plates with very thin cfrp faces." In 38th Structures, Structural Dynamics, and Materials Conference, p. 1249. 1997.

[5] Ko, William L. "Predictions of thermal buckling strengths of hypersonic aircraft sandwich panels using minimum potential energy and finite element methods." (1995).

[6] Babu, C. Sarath, and T. Kant. "Refined higher order finite element models for thermal buckling of laminated composite and sandwich plates." Journal of thermal stresses 23, no. 2 (2000): 111-130.

[7] Kant, T. A. R. U. N., and C. S. Babu. "Thermal buckling analysis of skew fibre-reinforced composite and sandwich plates using shear deformable finite element models." Composite Structures 49, no. 1 (2000): $77-85$.

[8] Shiau, Le-Chung, and Shih-Yao Kuo. "Thermal buckling of composite sandwich plates." Mechanics based design of structures and machines 32, no. 1 (2004): 57-72.

[9] Matsunaga, Hiroyuki. "Thermal buckling of cross-ply laminated composite and sandwich plates according to a global higher-order deformation theory." Composite Structures 68, no. 4 (2005): 439-454.

[10] Matsunaga, Hiroyuki. "Thermal buckling of angle-ply laminated composite and sandwich plates according to a global higher-order deformation theory." Composite structures 72, no. 2 (2006): 177-192.

[11] Vangipuram, Pradeep, and N. Ganesan. "Buckling and vibration of rectangular composite viscoelastic sandwich plates under thermal loads." Composite Structures 77, no. 4 (2007): 419-429.

[12] Pradeep, V., and N. Ganesan. "Thermal buckling and vibration behavior of multi-layer rectangular viscoelastic sandwich plates." Journal of Sound and Vibration 310, no. 1-2 (2008): 169-183.

[13] Han, Bin, Ke-Ke Qin, Qian-Cheng Zhang, Qi Zhang, Tian Jian Lu, and Bing-Heng Lu. "Free vibration and buckling of foam-filled composite corrugated sandwich plates under thermal loading." Composite Structures 172 (2017): 173-189.

[14] Vescovini, Riccardo, M. d'Ottavio, Lorenzo Dozio, and O. Polit. "Thermal buckling response of laminated and sandwich plates using refined 2-D models." Composite Structures 176 (2017): 313-328.

[15] Li, Jinqiang, Fengming Li, and Yoshihiro Narita. "Active control of thermal buckling and vibration for a sandwich composite laminated plate with piezoelectric fiber-reinforced composite actuator facesheets." Journal of Sandwich Structures \& Materials 21, no. 7 (2019): 2563-2581. 
[16] Zhai, Yanchun, Jianmin Su, and Sen Liang. "Free vibration and buckling analysis of composite sandwich plates in thermal environment." Journal of Sandwich Structures \& Materials (2018): 1099636218795375.

[17] Zhu, Jiang, Haiqing Peng, Fernando Rodriguez-Macias, John L. Margrave, Valery N. Khabashesku, Ashraf M. Imam, Karen Lozano, and Enrique V. Barrera. "Reinforcing epoxy polymer composites through covalent integration of functionalized nanotubes." Advanced Functional Materials 14, no. 7 (2004): 643648.

[18] Rahman, Muhammad M., Mahesh Hosur, Adriane G. Ludwick, Shaik Zainuddin, Ashok Kumar, Jonathan Trovillion, and Shaik Jeelani. "Thermo-mechanical behavior of epoxy composites modified with reactive polyol diluent and randomly-oriented amino-functionalized multi-walled carbon nanotubes." Polymer Testing 31, no. 6 (2012): 777-784.

[19] He, Yuxin, Song Yang, Hu Liu, Qian Shao, Qiuyu Chen, Chang Lu, Yuanli Jiang, Chuntai Liu, and Zhanhu Guo. "Reinforced carbon fiber laminates with oriented carbon nanotube epoxy nanocomposites: magnetic field assisted alignment and cryogenic temperature mechanical properties." Journal of colloid and interface science 517 (2018): 40-51.

[20] Maghsoudlou, Mohammad Ali, Reza Barbaz Isfahani, Saeed Saber-Samandari, and Mojtaba Sadighi. "Effect of interphase, curvature and agglomeration of SWCNTs on mechanical properties of polymer-based nanocomposites: Experimental and numerical investigations." Composites Part B: Engineering 175 (2019): 107119.

[21] Siddiqui, Naveed A., Man-Lung Sham, Ben Zhong Tang, Arshad Munir, and Jang-Kyo Kim. "Tensile strength of glass fibres with carbon nanotube-epoxy nanocomposite coating." Composites Part A: Applied Science and Manufacturing 40, no. 10 (2009): 1606-1614.

[22] Barai, Pallab, and George J. Weng. "A theory of plasticity for carbon nanotube reinforced composites." International Journal of Plasticity 27, no. 4 (2011): 539-559.

[23] Rahman, M. M., Sh Zainuddin, M. V. Hosur, J. E. Malone, M. B. A. Salam, Ashok Kumar, and S. Jeelani. "Improvements in mechanical and thermo-mechanical properties of e-glass/epoxy composites using amino functionalized MWCNTs." Composite Structures 94, no. 8 (2012): 2397-2406.

[24] Kamarian, S., M. Bodaghi, R. Barbaz Isfahani, M. Shakeri, and M. H. Yas. "Influence of carbon nanotubes on thermal expansion coefficient and thermal buckling of polymer composite plates: experimental and numerical investigations." Mechanics Based Design of Structures and Machines (2019): $1-16$.

[25] Sankar, A., S. Natarajan, T. Merzouki, and M. Ganapathi. "Nonlinear dynamic thermal buckling of sandwich spherical and conical shells with CNT reinforced facesheets." International Journal of Structural Stability and Dynamics 17, no. 09 (2017): 1750100.

[26] Ebrahimi, Farzad, and Navid Farazmandnia. "Thermal buckling analysis of functionally graded carbon nanotube-reinforced composite sandwich beams." Steel and composite structures 27, no. 2 (2018): 149159.

[27] Mehar, Kulmani, Subrata Kumar Panda, Yuvarajan Devarajan, and Gautam Choubey. "Numerical buckling analysis of graded CNT-reinforced composite sandwich shell structure under thermal loading." Composite Structures 216 (2019): 406-414. 
[28] Van Tung, Hoang, and Vu Thanh Long. "Buckling and postbuckling of CNT-reinforced composite sandwich cylindrical panels subjected to axial compression in thermal environments." Vietnam Journal of Mechanics 41, no. 3 (2019): 217-231.

[29] Malekzadeh, P., and M. Shojaee. "Buckling analysis of quadrilateral laminated plates with carbon nanotubes reinforced composite layers." Thin-Walled Structures 71 (2013): 108-118.

[30] Malekzadeh, P., and A. R. Zarei. "Free vibration of quadrilateral laminated plates with carbon nanotube reinforced composite layers." Thin-Walled Structures 82 (2014): 221-232.

[31] Hyer, Michael W., and Scott R. White. Stress analysis of fiber-reinforced composite materials. DEStech Publications, Inc, 2009.

[32] Li, Xiangyang, Kaiping Yu, Jingyong Han, Rui Zhao, and Ying Wu. "A piecewise shear deformation theory for free vibration of composite and sandwich panels." Composite Structures 124 (2015): 111-119.

[33] Li, Xiangyang, Kaiping Yu, and Rui Zhao. "Thermal post-buckling and vibration analysis of a symmetric sandwich beam with clamped and simply supported boundary conditions." Archive of Applied Mechanics 88, no. 4 (2018): 543-561.

[34] Reddy, Junuthula Narasimha. Mechanics of laminated composite plates and shells: theory and analysis. CRC press, 2003.

[35] Shu, Chang, and Bryan E. Richards. "Application of generalized differential quadrature to solve twodimensional incompressible Navier-Stokes equations." International Journal for Numerical Methods in Fluids 15, no. 7 (1992): 791-798.

[36] Tornabene, Francesco, Nicholas Fantuzzi, Francesco Ubertini, and Erasmo Viola. "Strong formulation finite element method based on differential quadrature: a survey." Applied Mechanics Reviews 67, no. 2 (2015). 


\section{Appendix 1}

$$
\begin{aligned}
& \bar{Q}_{11}=Q_{11} \cos ^{4} \theta+2\left(Q_{12}+2 Q_{66}\right) \operatorname{Sin}^{2} \theta \operatorname{Cos}^{2} \theta+Q_{22} \operatorname{Sin}^{4} \theta \\
& \bar{Q}_{12}=\left(Q_{11}+Q_{22}-4 Q_{66}\right) \operatorname{Sin}^{2} \theta \operatorname{Cos}^{2} \theta+Q_{12}\left(\operatorname{Sin}^{4} \theta+\operatorname{Cos}^{4} \theta\right) \\
& \bar{Q}_{22}=Q_{11} \operatorname{Sin}^{4} \theta+2\left(Q_{12}+2 Q_{66}\right) \operatorname{Sin}^{2} \theta \operatorname{Cos}^{2} \theta+Q_{22} \operatorname{Cos}^{4} \theta \\
& \bar{Q}_{16}=\left(Q_{11}-Q_{12}-2 Q_{66}\right) \operatorname{Sin} \theta \operatorname{Cos}^{3} \theta+\left(Q_{12}-Q_{22}+2 Q_{66}\right) \operatorname{Sin}^{3} \theta \operatorname{Cos} \theta \\
& \bar{Q}_{26}=\left(Q_{11}-Q_{12}-2 Q_{66}\right) \operatorname{Sin}^{3} \theta \operatorname{Cos} \theta+\left(Q_{12}-Q_{22}+2 Q_{66}\right) \operatorname{Sin} \theta \operatorname{Cos}^{3} \theta \\
& \bar{Q}_{66}=\left(Q_{11}+Q_{22}-2 Q_{12}-2 Q_{66}\right) \operatorname{Sin}^{2} \theta \operatorname{Cos}^{2} \theta+Q_{66}\left(\operatorname{Sin}^{4} \theta+\operatorname{Cos}^{4} \theta\right) \\
& \bar{Q}_{44}=Q_{44} \operatorname{Cos}^{2} \theta+Q_{55} \operatorname{Sin}^{2} \theta \\
& \bar{Q}_{45}=\left(Q_{55}-Q_{44}\right) \operatorname{Cos} \theta \operatorname{Sin} \theta \\
& \bar{Q}_{55}=Q_{44} \operatorname{Sin}^{2} \theta+Q_{55} \operatorname{Cos}^{2} \theta
\end{aligned}
$$

where $\theta$ is the angle that the fibers of $k^{\text {th }}$ lamina makes with the structure x-axis, and $Q_{i j}$, called the plane stress-reduced stiffnesses, are given by [34]:

$$
\begin{aligned}
Q_{11} & =\frac{E_{1}}{1-v_{12} v_{21}} \\
Q_{12} & =\frac{v_{12} E_{2}}{1-v_{12} v_{21}}=\frac{v_{21} E_{1}}{1-v_{12} v_{21}} \\
Q_{22} & =\frac{E_{2}}{1-v_{12} v_{21}} \\
Q_{44} & =G_{23} \\
Q_{55} & =G_{13} \\
Q_{66} & =G_{12}
\end{aligned}
$$

Where, $E_{1}, E_{2}, v_{12}$ and $G_{12}$ are the independent material constants. 


\section{Appendix 2}

$\alpha_{x}=\alpha_{1} \cos ^{2} \theta+\alpha_{2} \operatorname{Sin}^{2} \theta$

$\alpha_{y}=\alpha_{1} \sin ^{2} \theta+\alpha_{2} \cos ^{2} \theta$

$\alpha_{x y}=2\left(\alpha_{1}-\alpha_{2}\right) \operatorname{Sin} \theta \cos \theta$

where $\alpha_{1}$ and $\alpha_{2}$ are the longitudinal and transverse coefficients of thermal expansion of the composite material. 


\section{Appendix 3}

$$
\begin{aligned}
& g_{11}^{(1)}=2\left(\frac{2 h_{c}+h_{f}}{2}\right)^{2} A_{11}^{f}+\left(\frac{2 h_{c}+h_{f}}{2 h_{c}}\right)^{2} D_{11}^{c} \\
& g_{22}^{(1)}=2\left(\frac{2 h_{c}+h_{f}}{2}\right)^{2} A_{22}^{f}+\left(\frac{2 h_{c}+h_{f}}{2 h_{c}}\right)^{2} D_{22}^{c} \\
& g_{12}^{(1)}=2\left(\frac{2 h_{c}+h_{f}}{2}\right)^{2} A_{12}^{f}+\left(\frac{2 h_{c}+h_{f}}{2 h_{c}}\right)^{2} D_{12}^{c} \\
& g_{66}^{(1)}=2\left(\frac{2 h_{c}+h_{f}}{2}\right)^{2} A_{66}^{f}+\left(\frac{2 h_{c}+h_{f}}{2 h_{c}}\right)^{2} D_{66}^{c} \\
& g_{11}^{(2)}=\left(\frac{h_{f}}{2 h_{c}}\right)\left(\frac{2 h_{c}+h_{f}}{2 h_{c}}\right) D_{11}^{c} \\
& g_{22}^{(2)}=\left(\frac{h_{f}}{2 h_{c}}\right)\left(\frac{2 h_{c}+h_{f}}{2 h_{c}}\right) D_{22}^{c} \\
& g_{12}^{(2)}=\left(\frac{h_{f}}{2 h_{c}}\right)\left(\frac{2 h_{c}+h_{f}}{2 h_{c}}\right) D_{12}^{c} \\
& g_{66}^{(2)}=\left(\frac{h_{f}}{2 h_{c}}\right)\left(\frac{2 h_{c}+h_{f}}{2 h_{c}}\right) D_{66}^{c} \\
& g_{11}^{(3)}=2 D_{11}^{f}+\left(\frac{h_{f}}{2 h_{c}}\right)^{2} D_{11}^{c} \\
& g_{22}^{(3)}=2 D_{22}^{f}+\left(\frac{h_{f}}{2 h_{c}}\right)^{2} D_{22}^{c} \\
& g_{12}^{(3)}=2 D_{12}^{f}+\left(\frac{h_{f}}{2 h_{c}}\right)^{2} D_{12}^{c} \\
& g_{66}^{(3)}=2 D_{66}^{f}+\left(\frac{h_{f}}{2 h_{c}}\right)^{2} D_{66}^{c}
\end{aligned}
$$




\section{Appendix 4}

$$
\begin{aligned}
& k_{11}=\left(\frac{m \pi}{a}\right)^{4} g_{11}^{(3)}+\left(\frac{m \pi}{a}\right)^{2}\left(\frac{n \pi}{b}\right)^{2}\left(2 g_{12}^{(3)}+4 g_{66}^{(3)}\right)+\left(\frac{n \pi}{b}\right)^{4} g_{22}^{(3)}+\left(\frac{m \pi}{a}\right)^{2}\left(S_{c x z}-N_{x}^{T}\right)+\left(\frac{n \pi}{b}\right)^{2}\left(S_{c y z}-N_{y}^{T}\right) \\
& k_{12}=\left(\frac{m \pi}{a}\right)^{3} g_{11}^{(2)}+\left(\frac{m \pi}{a}\right)\left(\frac{n \pi}{b}\right)^{2}\left(g_{12}^{(2)}+2 g_{66}^{(2)}\right)-\left(\frac{m \pi}{a}\right) S_{c x z} \\
& k_{13}=\left(\frac{n \pi}{b}\right)^{3} g_{22}^{(2)}+\left(\frac{m \pi}{a}\right)^{2}\left(\frac{n \pi}{b}\right)\left(g_{12}^{(2)}+2 g_{66}^{(2)}\right)-\left(\frac{n \pi}{b}\right) S_{c y z} \\
& k_{21}=k_{12} \\
& k_{22}=\left(\frac{m \pi}{a}\right)^{2} g_{11}^{(1)}+\left(\frac{n \pi}{b}\right)^{2} g_{66}^{(1)}+S_{c x z} \\
& k_{23}=\left(\frac{m \pi}{a}\right)\left(\frac{n \pi}{b}\right)\left(g_{12}^{(1)}+g_{66}^{(1)}\right) \\
& k_{31}=k_{13} \\
& k_{32}=k_{23} \\
& k_{33}=\left(\frac{n \pi}{b}\right)^{2} g_{22}^{(1)}+\left(\frac{m \pi}{a}\right)^{2} g_{66}^{(1)}+S_{c y z}
\end{aligned}
$$




\section{Appendix 5}

$$
\begin{aligned}
& p_{1}=g_{11}^{(3)} \\
& p_{2}=-\left(\frac{n \pi}{b}\right)^{2}\left(2 g_{12}^{(3)}+4 g_{66}^{(3)}\right)+\left(N_{x}^{T}-S_{c x z}\right) \\
& P_{3}=\left(\frac{n \pi}{b}\right)^{4} g_{22}^{(3)}+\left(\frac{n \pi}{b}\right)^{2}\left(S_{c y z}-N_{y y}^{T}\right) \\
& P_{4}=g_{11}^{(2)} \\
& P_{5}=-\left(\frac{n \pi}{b}\right)^{2}\left(g_{12}^{(2)}+2 g_{66}^{(2)}\right)+S_{c x z} \\
& P_{6}=-\left(\frac{n \pi}{b}\right)\left(g_{12}^{(2)}+2 g_{66}^{(2)}\right) \\
& p_{7}=\left(\frac{n \pi}{b}\right)^{3} g_{22}^{(2)}-\left(\frac{n \pi}{b}\right) S_{c y z} \\
& p_{8}=-g_{11}^{(2)} \\
& p_{9}=\left(\frac{n \pi}{b}\right)^{2}\left(g_{12}^{(2)}+2 g_{66}^{(2)}\right)-S_{c x z} \\
& p_{10}=-g_{11}^{(1)} \\
& p_{11}=\left(\frac{n \pi}{b}\right)^{2} g_{66}^{(1)}+S_{c x z} \\
& p_{12}=\left(\frac{n \pi}{b}\right)\left(g_{12}^{(1)}+g_{66}^{(1)}\right) \\
& p_{13}=-\left(\frac{n \pi}{b}\right)\left(g_{12}^{(2)}+2 g_{66}^{(2)}\right) \\
& p_{14}=\left(\frac{n \pi}{b}\right)^{3} g_{22}^{(2)}-\left(\frac{n \pi}{b}\right) S_{c y z} \\
& p_{15}=-\left(\frac{n \pi}{b}\right)\left(g_{12}^{(1)}+g_{66}^{(1)}\right) \\
& p_{16}=-g_{66}^{(1)} \\
& p_{17}=\left(\frac{n \pi}{b}\right)^{2} g_{22}^{(1)}+S_{c y z}
\end{aligned}
$$




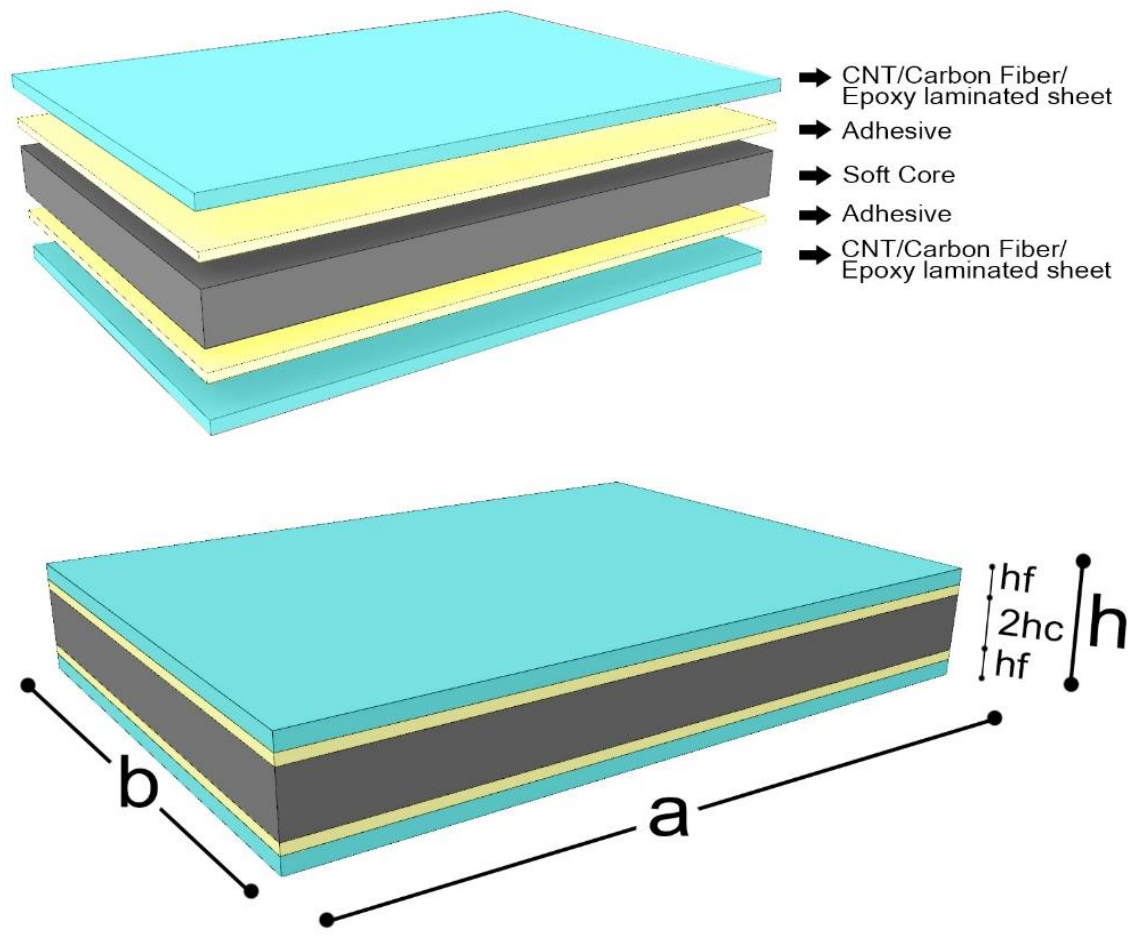

Fig 1 Schematic of rectangular sandwich plates with hybrid nanocomposite face sheets 


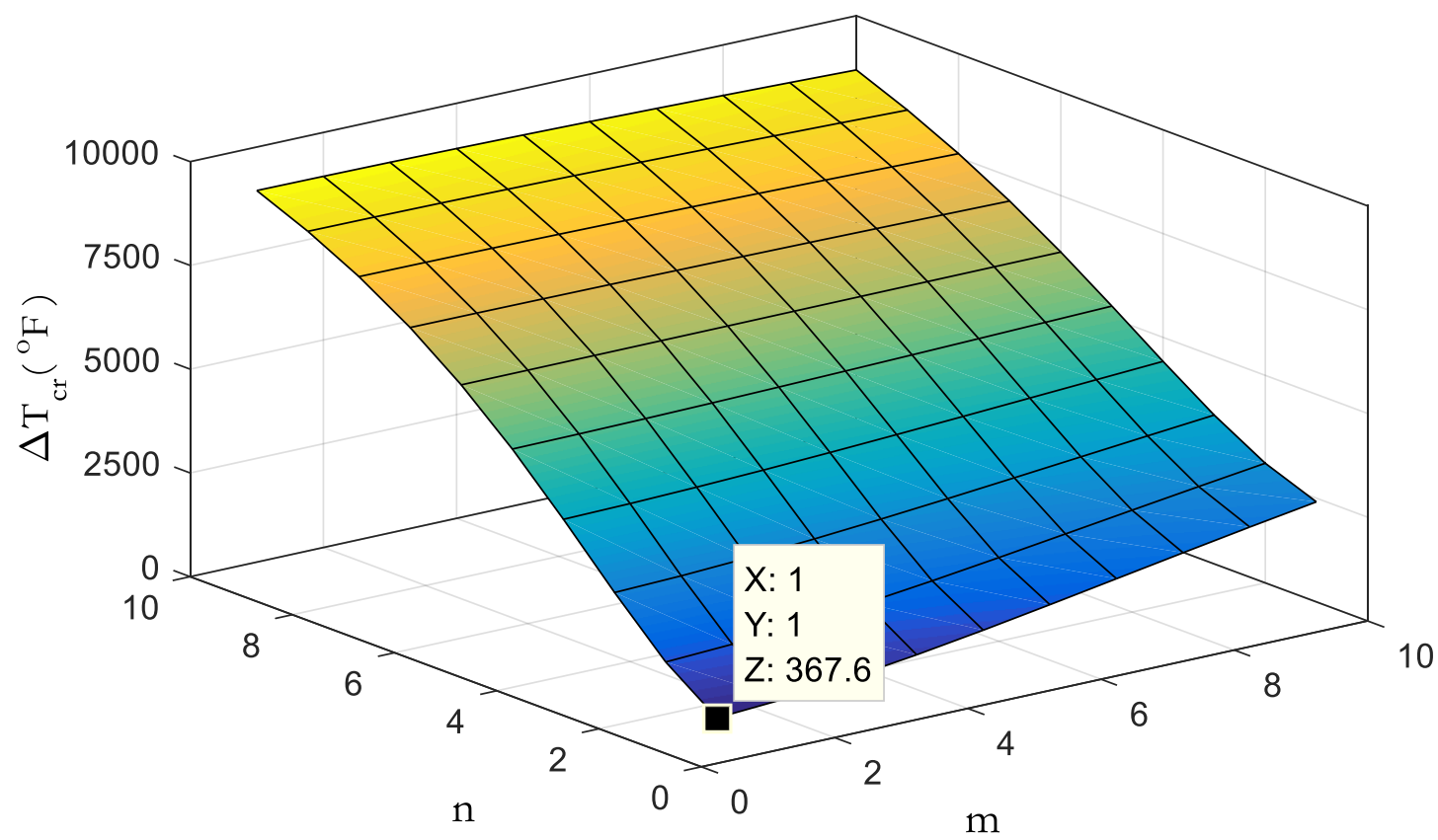

Fig 2 Buckling temperature of simply supported sandwich plates with aluminium honeycomb core and titanium face sheets relative to half-wave numbers ( $a=24$ in $, b / a=3, h=0.81$ in,$h_{f}=0.06$ in ) 


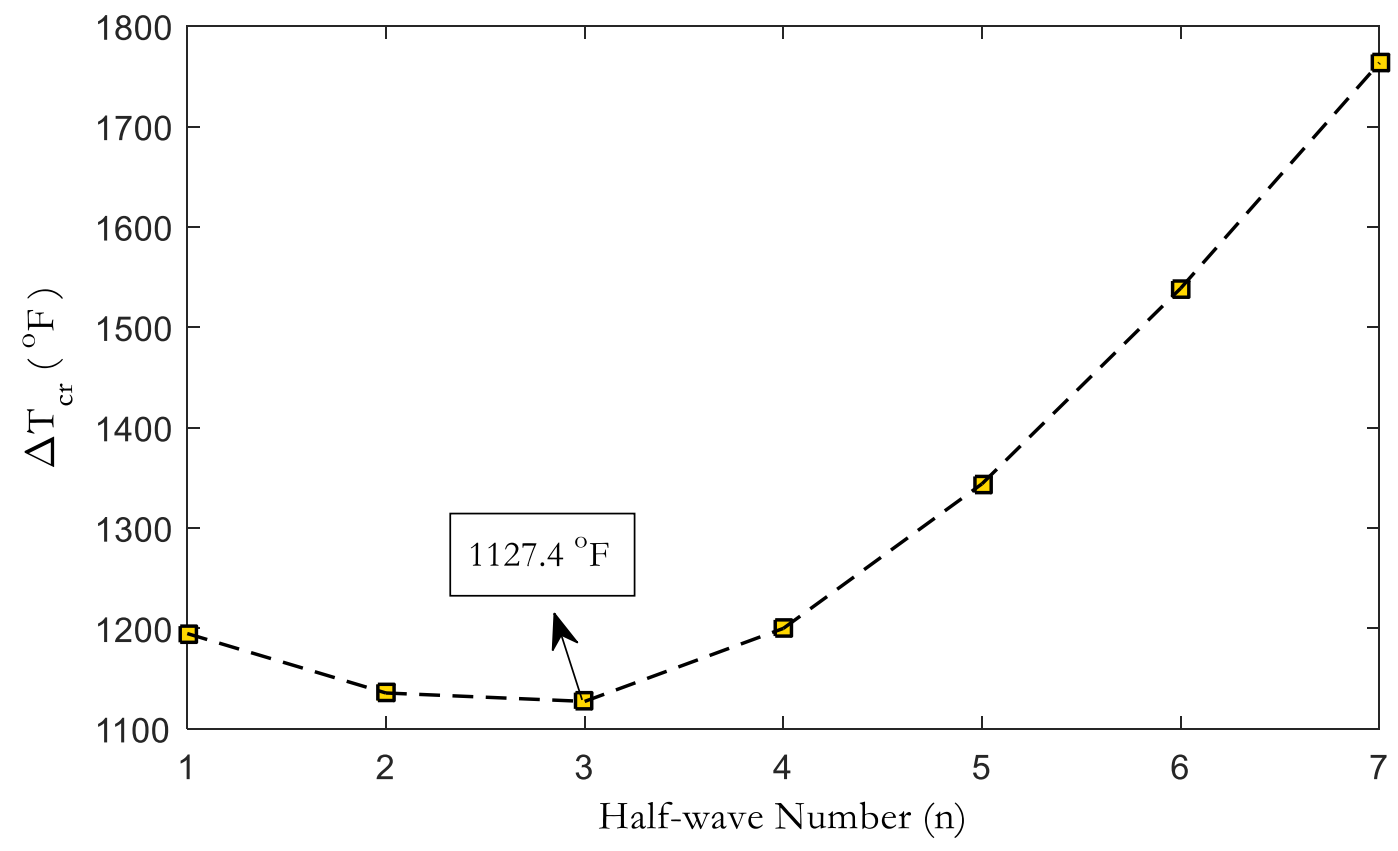

Fig 3 Buckling temperature of CSCS supported sandwich plates with aluminium honeycomb core and titanium face sheets relative to half-wave numbers ( $a=24 \mathrm{in}, b / a=3, h=0.81 \mathrm{in}, h_{f}=0.06 \mathrm{in}$ ) 


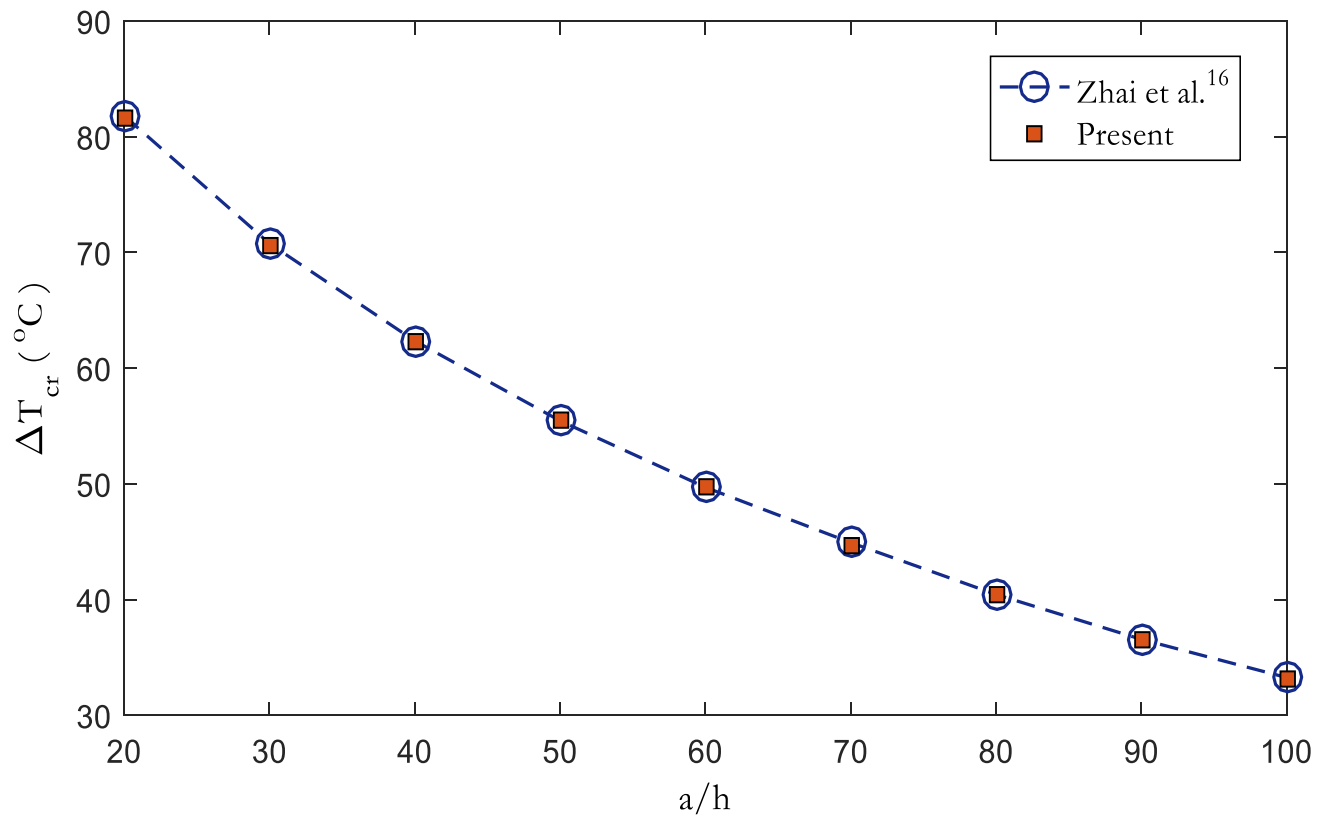

Fig 4 Critical buckling temperature of a simply supported sandwich plate with soft core and single-layer orthotropic skins $\left(a=b=0.3 \mathrm{~m}, h_{f}=0.1 \mathrm{~mm}\right)$ 

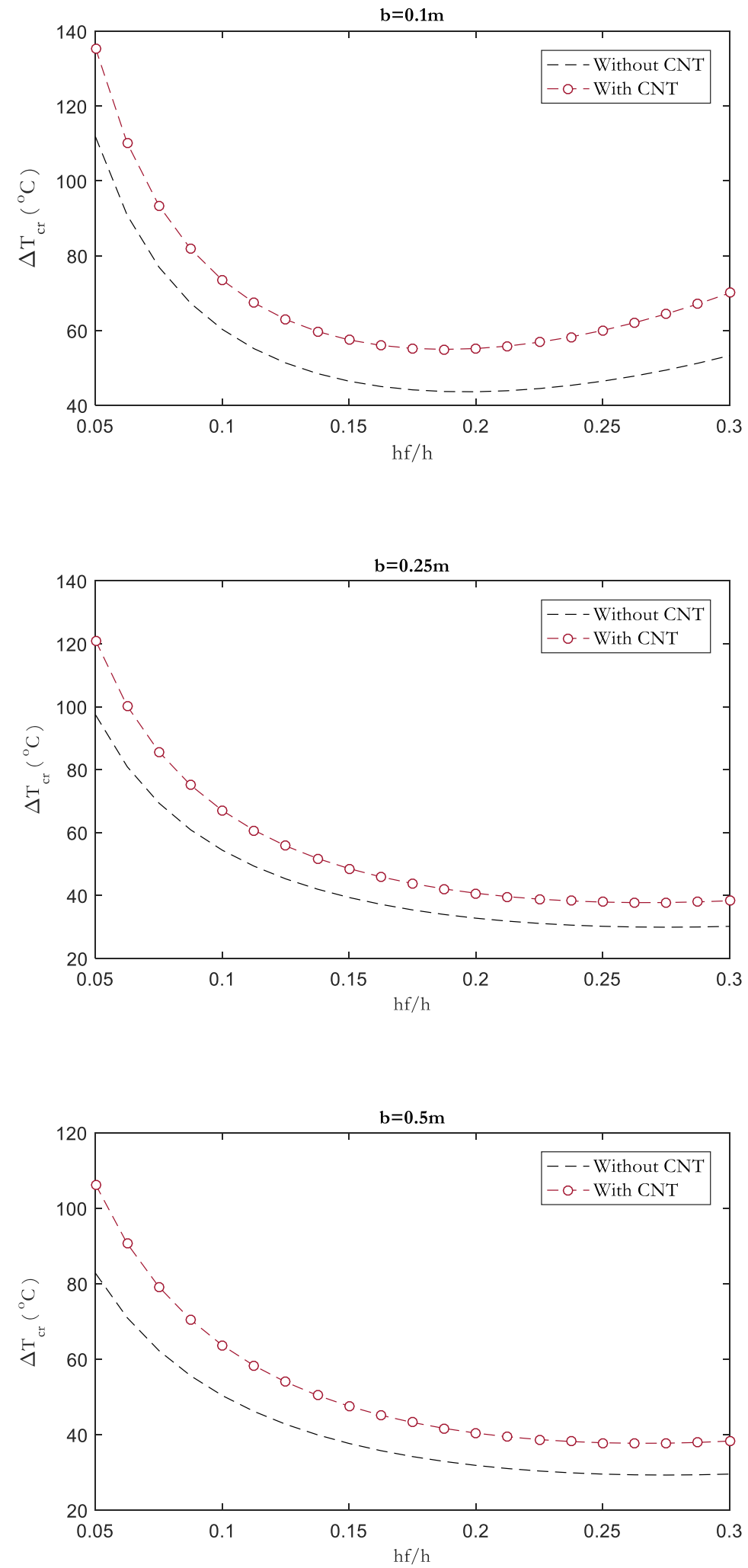

Fig 5 Effect of CNTs on the critical buckling temperature of simply supported sandwich plates for various geometrical parameters $(a=0.5 m, a / h=50)$ 

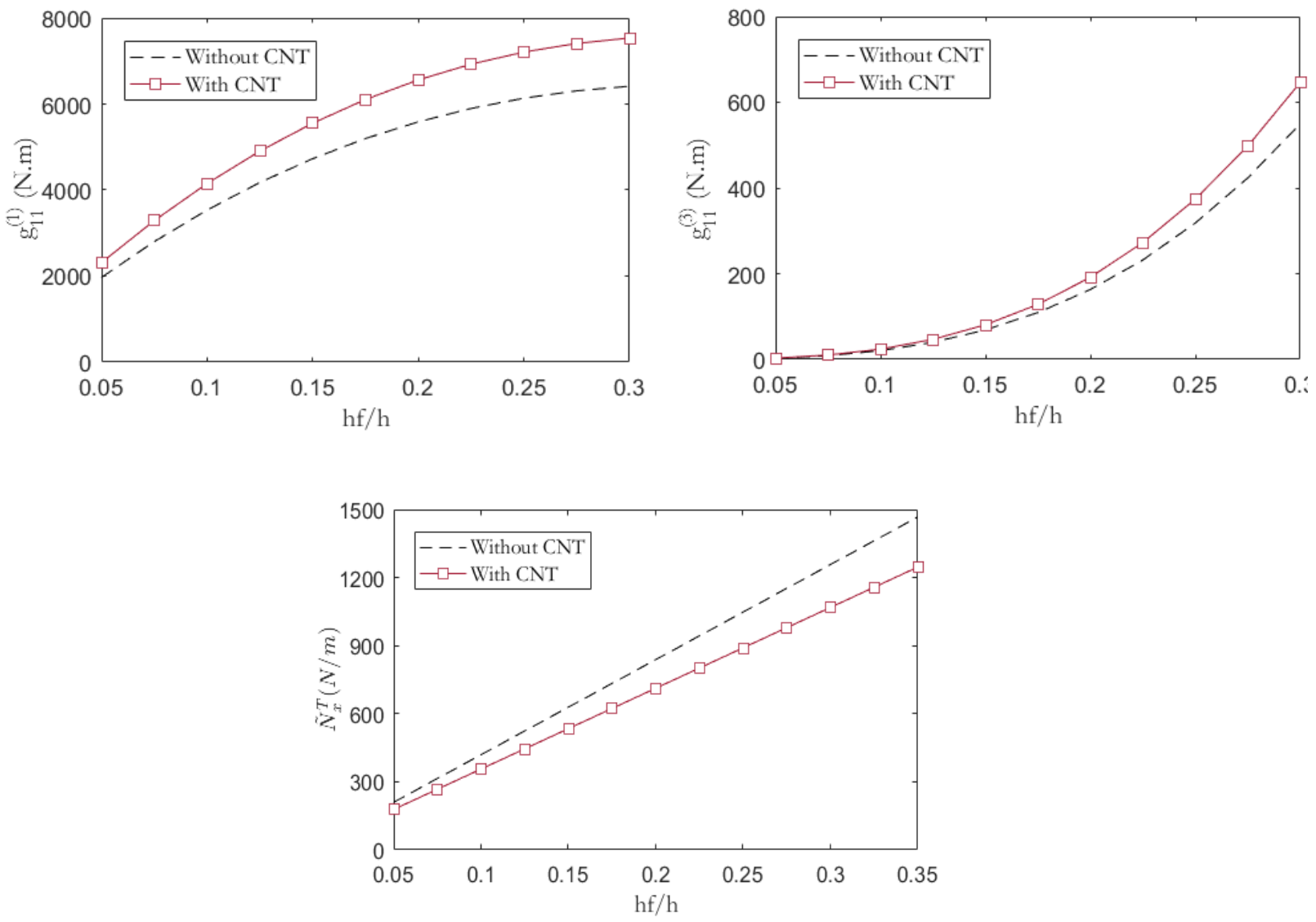

Fig 6 Effect of CNTs on the stiffness coefficients and in-plane thermal force in sandwich plates for various values of $h_{f} / h$ ratios $(a=b=0.5 m, a / h=50)$ 


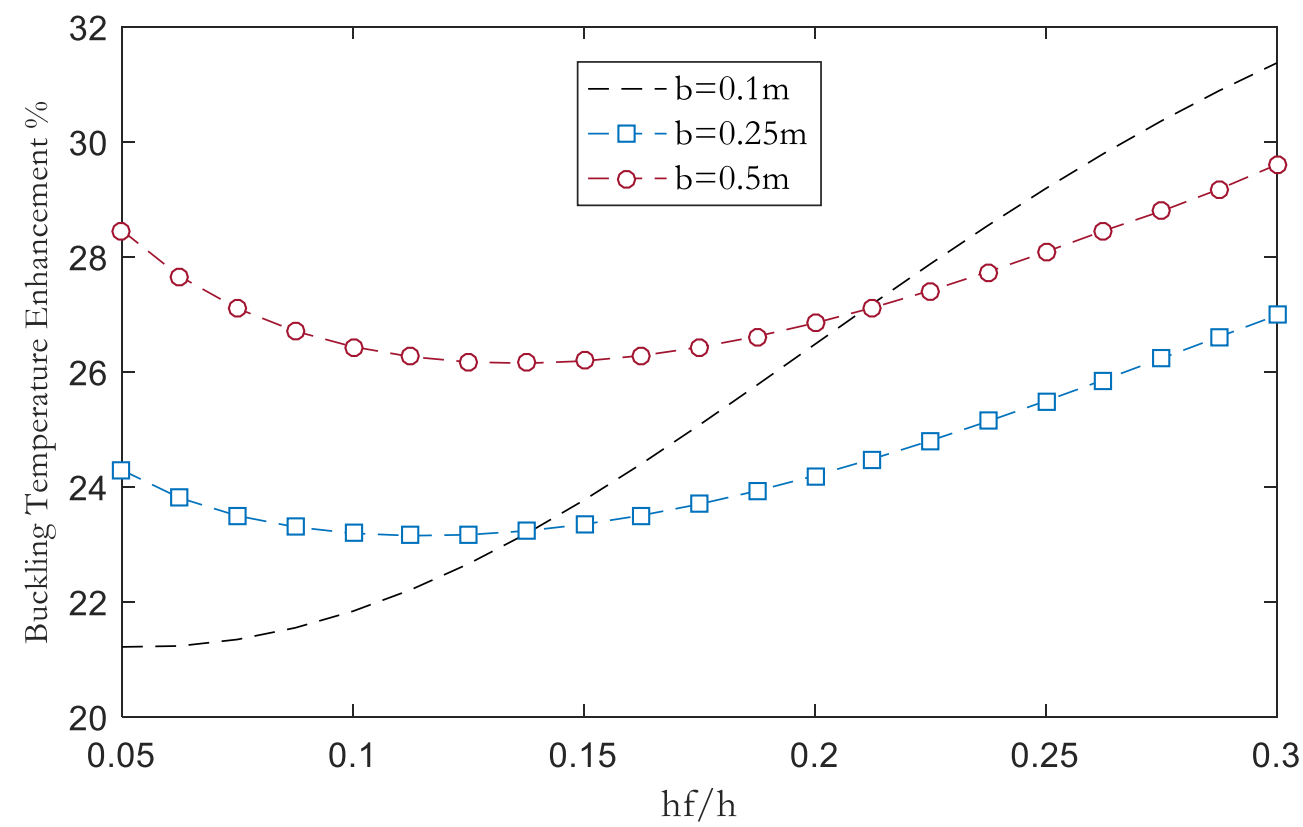

Fig 7 Increases in the buckling temperature of simply supported sandwich plates given the use of CNTs in the face sheets for various geometrical parameters $(a=0.5 m, a / h=50)$ 


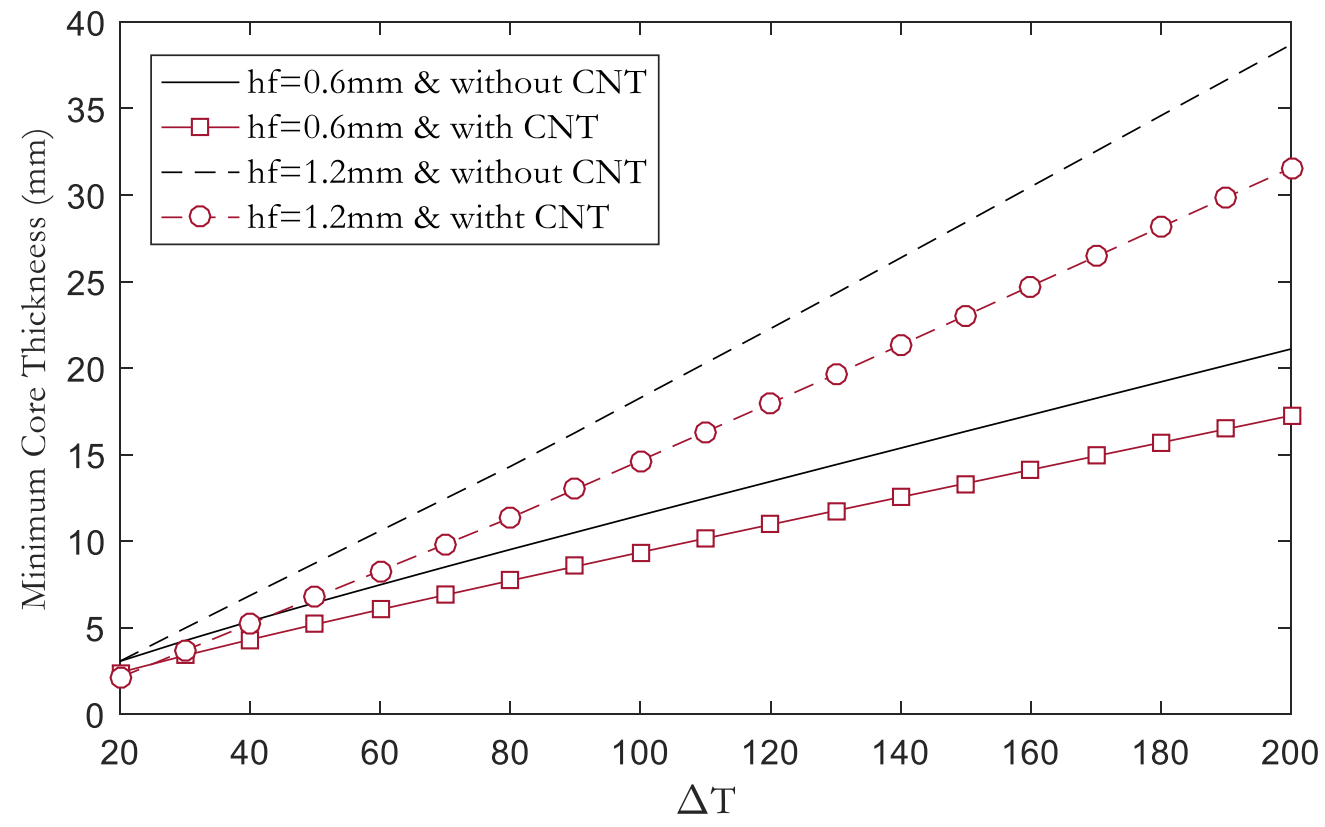

Fig 8 Effect of CNTs on the minimum core thickness ( $2 h c$ ) necessary to prevent thermal buckling phenomenon in the square sandwich plate with simply supported boundary conditions

$$
(a=0.5 m, b=0.5 m)
$$



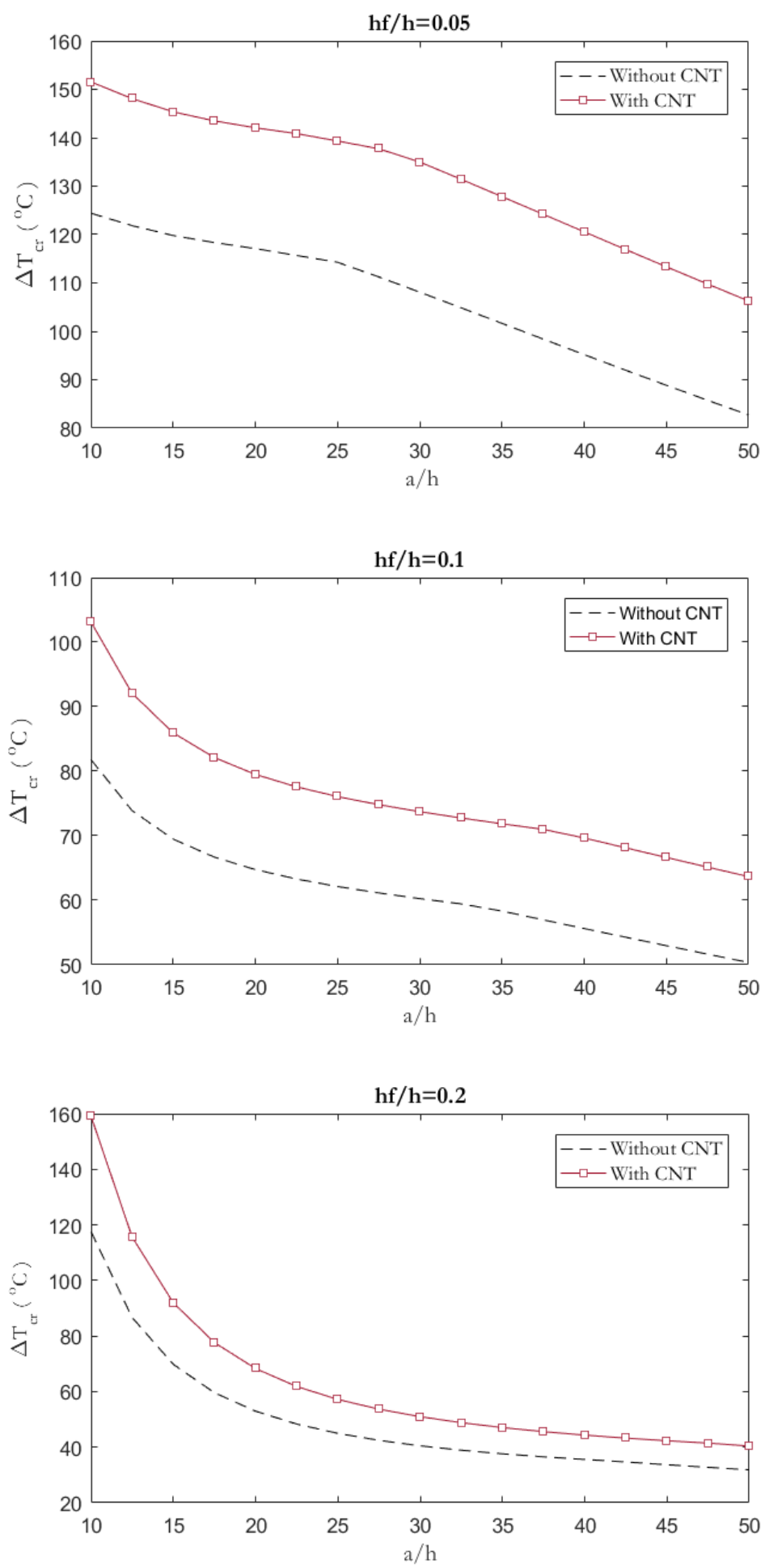

Fig 9 Effect of CNTs on the critical buckling temperature of simply supported square sandwich plates with respect to $a / h$ ratio for different values of the $h_{f} / h$ parameter 


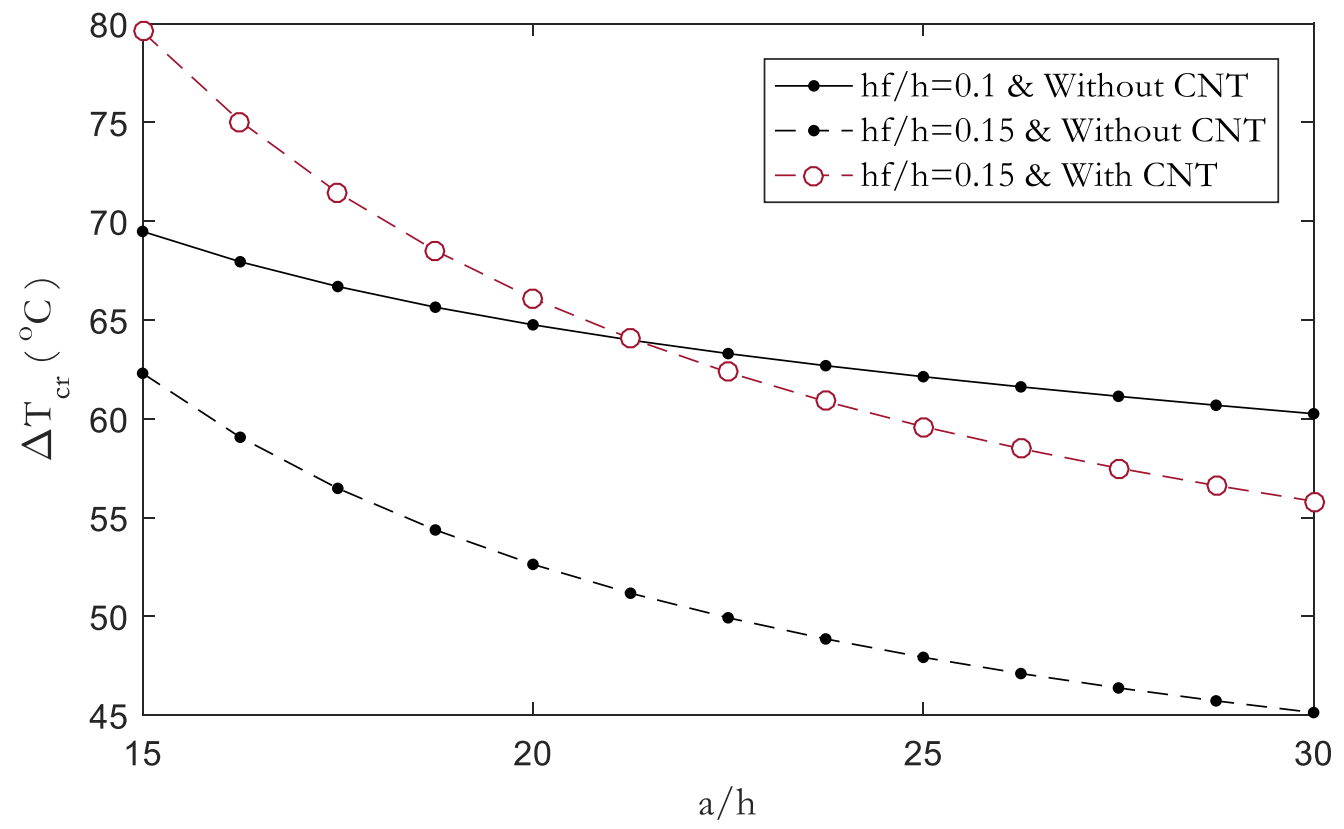

Fig 10 Effect of using CNTs on critical buckling temperature of simply supported square sandwich plates relative to the $a / h$ ratio 


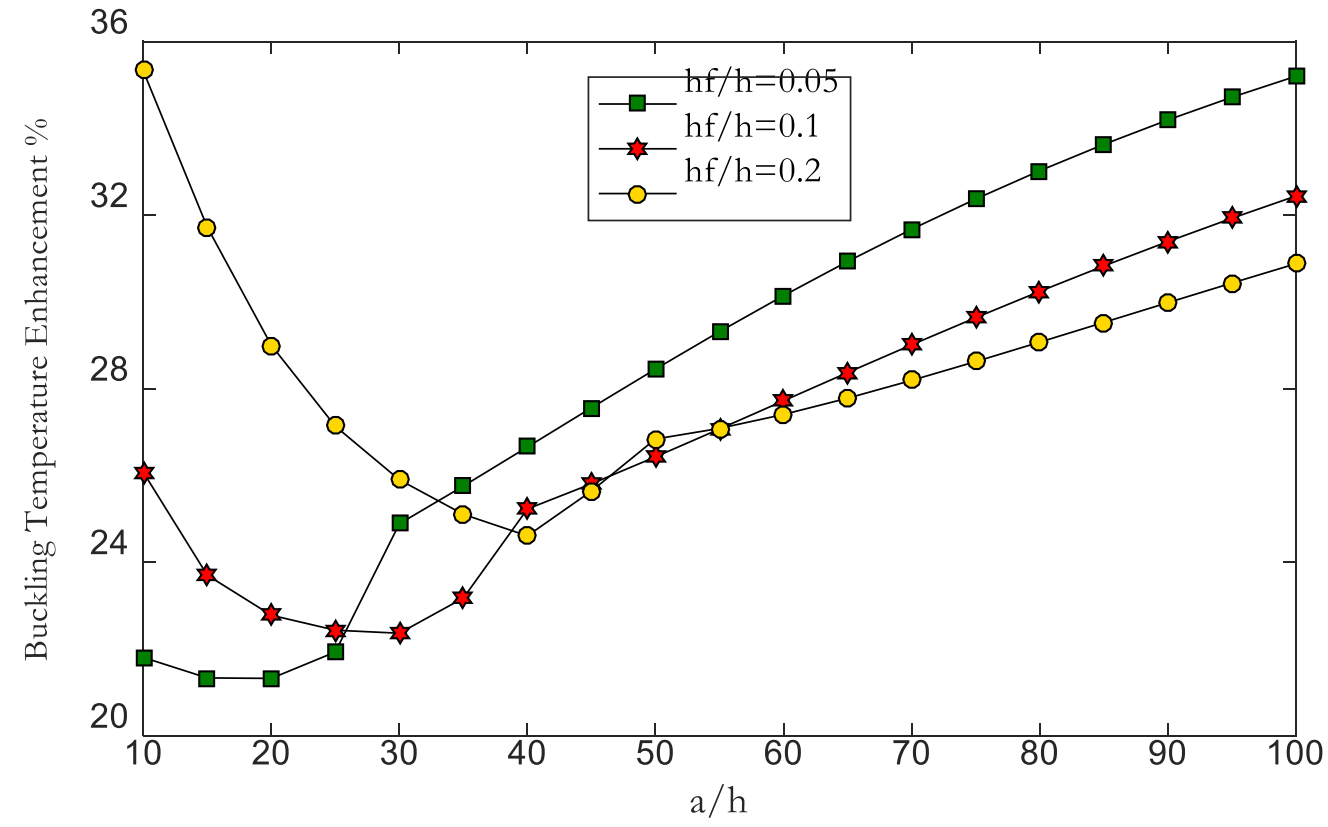

Fig 11 Increases in the buckling temperature of simply supported sandwich plates by using CNTs in the face sheets relative to $a / h$ ratio for different values of the $h_{f} / h$ parameter 


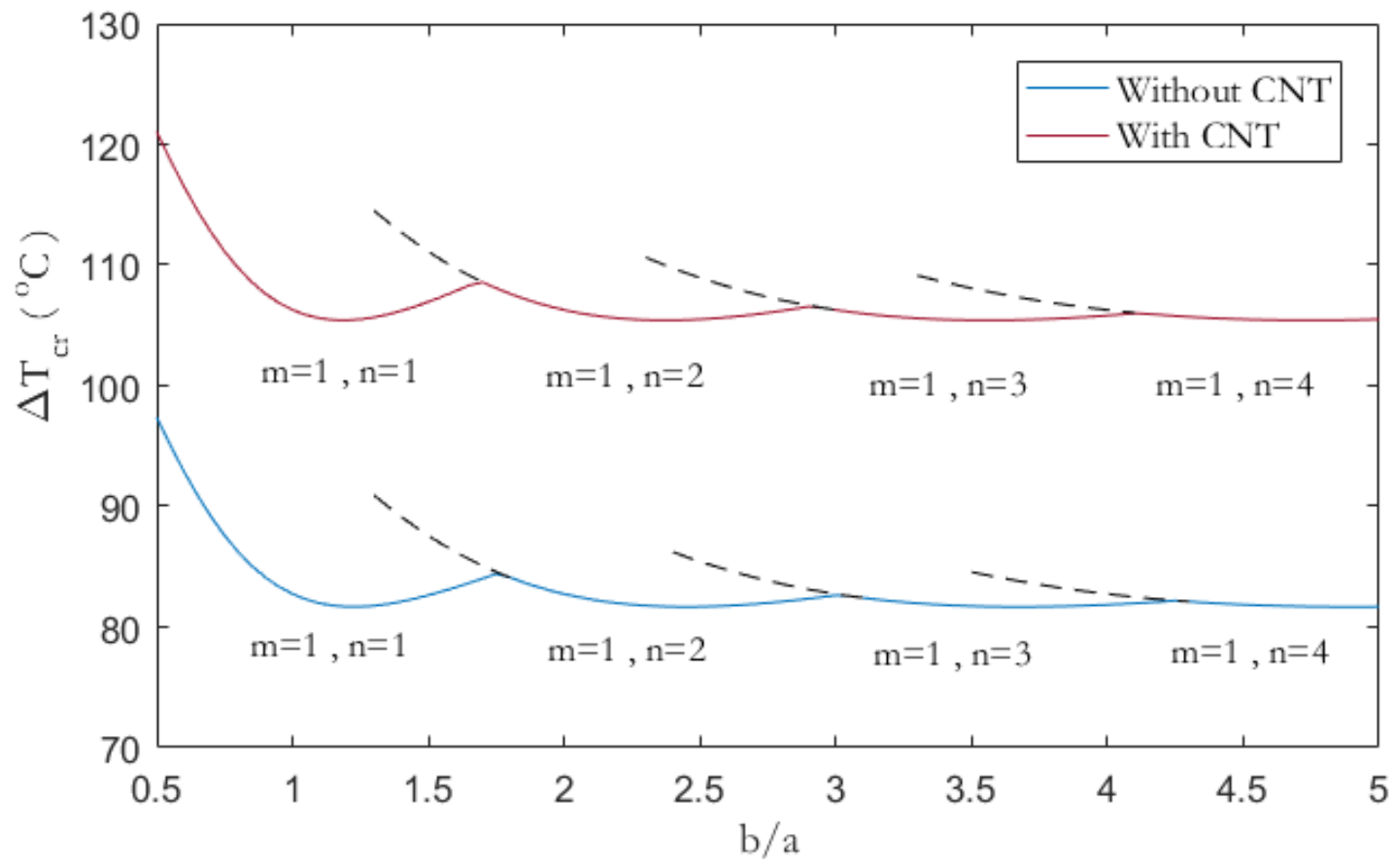

Fig 12 Effect of CNTs on the buckling temperature of simply supported sandwich plates relative to the b/a ratio $\left(a=0.5 \mathrm{~m}, h_{f} / h=0.05, a / h=50\right)$ 


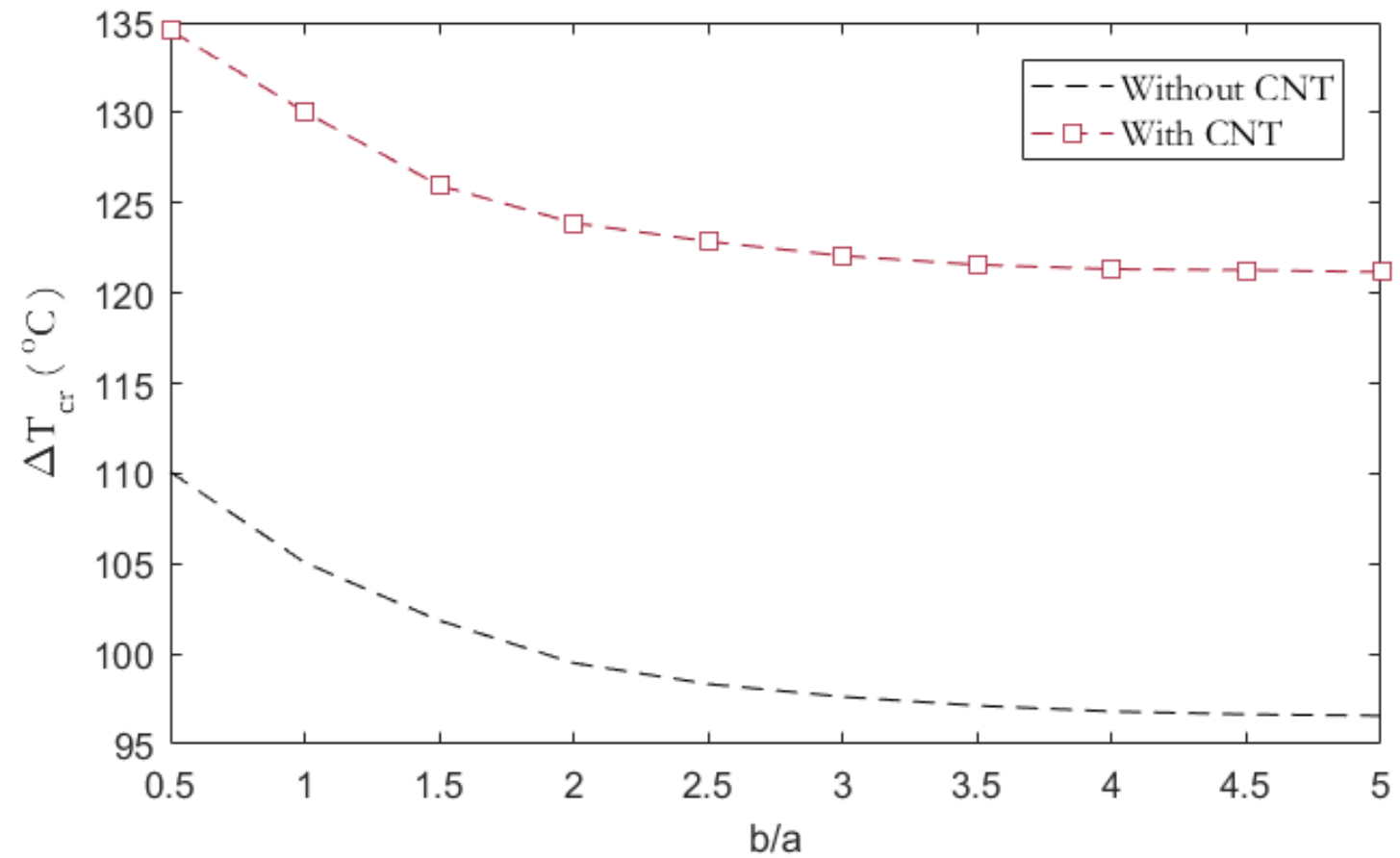

Fig 13 Effect of CNTs on the buckling temperature of fully clamped sandwich plates relative to the $b / a$ ratio

$\left(a=0.5 m, h_{f} / h=0.05, a / h=50\right)$ 


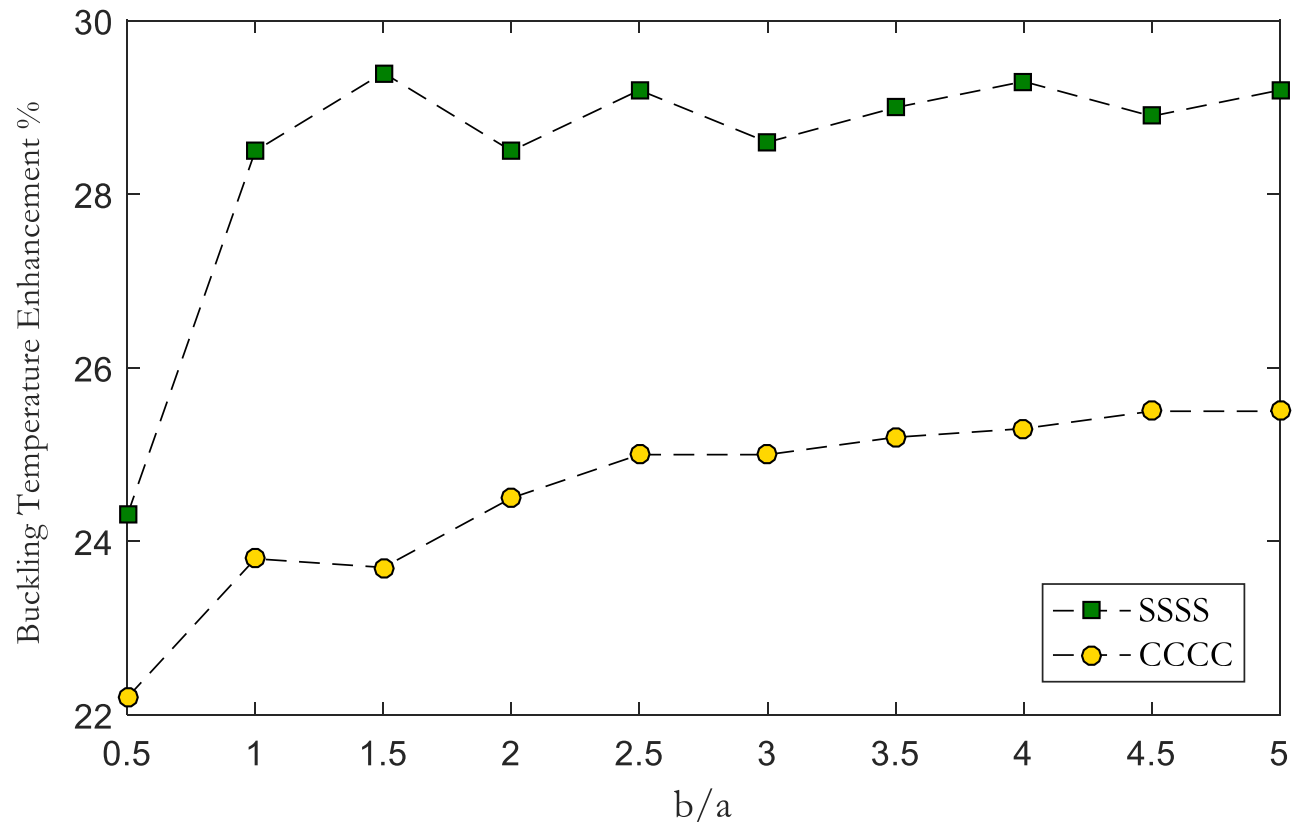

Fig 14 Increases in buckling temperature of sandwich plates by using CNTs in face sheets for different aspect ratios and boundary conditions $\left(a=0.5 \mathrm{~m}, h_{f} / h=0.05, a / h=50\right)$ 
Table 1 Influence of CNTs on thermo-mechanical properties of carbon fiber/epoxy composites

\begin{tabular}{cccc}
\hline Property & Unit & Carbon fiber/epoxy & Carbon fiber/CNT/epoxy \\
\hline$E_{1}$ & $G P a$ & 125.8 & 147.9 \\
$E_{2}$ & $G P a$ & 8.7 & 10.0 \\
$G_{12}$ & $G P a$ & 4.1 & 4.8 \\
$v_{12}$ & - & 0.29 & 0.29 \\
$\alpha_{1}$ & $1 /^{\circ} C$ & $0.4 \times 10^{-6}$ & $0.2 \times 10^{-6}$ \\
$\alpha_{2}$ & $1 /^{\circ} C$ & $47.9 \times 10^{-6}$ & $35.2 \times 10^{-6}$ \\
\hline
\end{tabular}

Table 2 Convergence study for GDQ approach in predicting the critical buckling temperatures of sandwich plates with $\left[0^{\circ} / 90^{\circ} / 0^{\circ}\right]$ nanocomposite face sheets and two different boundary conditions

\begin{tabular}{|c|c|c|c|}
\hline \multicolumn{2}{|c|}{ CSCS } & \multicolumn{2}{|c|}{ CCCC } \\
\hline$N_{x}$ & $\Delta T_{c r}\left({ }^{o} \mathrm{C}\right)$ & $N_{x} \times N_{y}$ & $\Delta T_{c r}\left({ }^{o} C\right)$ \\
\hline 5 & 121.0 & $5 \times 5$ & $131 . .8$ \\
\hline 7 & 120.7 & $7 \times 7$ & 129.2 \\
\hline 9 & 120.7 & $9 \times 9$ & 130.0 \\
\hline 13 & 120.7 & $13 \times 13$ & 130.0 \\
\hline 17 & 120.7 & $17 \times 17$ & 130.0 \\
\hline 21 & 120.7 & $21 \times 21$ & 130.0 \\
\hline
\end{tabular}


Table 3 Verification study for analytical, semi-analytical and numerical methods in critical buckling temperature $\left({ }^{\circ} \mathrm{F}\right)$ estimation of sandwich plates with aluminum honeycomb core and titanium face sheets

$$
\text { ( } \left.a=24 \text { in }, h=0.81 \text { in, } h_{f}=0.06 \text { in }\right)
$$

\begin{tabular}{|c|c|c|c|c|c|c|}
\hline \multirow{2}{*}{$\begin{array}{c}\text { Boundary } \\
\text { Conditions }\end{array}$} & \multirow{2}{*}{$b / a$} & \multicolumn{2}{|c|}{ Present } & \multicolumn{2}{|c|}{$\mathrm{Ko}^{5}$} & \multirow{2}{*}{ Shiau and $\mathrm{Kuo}^{8}$} \\
\hline & & Solution & & Energy method & FE method & \\
\hline \multirow[t]{4}{*}{ SSSS } & 1 & Analytical & $627.5(1,1)^{*}$ & 622 & 583 & 631 \\
\hline & 2 & & $409.9(1,1)$ & 409 & 403 & 411 \\
\hline & 3 & & $367.6(1,1)$ & 368 & 366 & 368 \\
\hline & 4 & & $352.6(1,1)$ & 358 & 348 & 353 \\
\hline \multirow[t]{4}{*}{ CSCS } & 1 & Semi-Analytical & $1127.4(1)^{* *}$ & 1128 & 1093 & - \\
\hline & 2 & & $1127.4(2)$ & 1128 & 1096 & - \\
\hline & 3 & & $1127.4(3)$ & 1128 & 1095 & - \\
\hline & 4 & & $1126.8(3)$ & 1126 & 1100 & - \\
\hline \multirow[t]{4}{*}{$\mathrm{CCCC}$} & 1 & Numerical & 1442.0 & 1428 & 1396 & 1456 \\
\hline & 2 & & 1181.2 & 1175 & 1160 & 1173 \\
\hline & 3 & & 1152.0 & 1150 & 1145 & 1148 \\
\hline & 4 & & 1147.2 & 1140 & 1121 & 1136 \\
\hline
\end{tabular}

* The numbers in the parenthesis show half waves numbers $(m, n)$ in Eq. (15) for analytical solution.

**The number in the parenthesis denotes half wave number $(n)$ in Eq. (20) for semi-analytical solution.

Table 4 Critical buckling temperature parameter $\left(\lambda_{T}=100 \alpha_{0} \Delta T_{c r}\right)$ of cross-ply $\left[0^{\circ} / 90^{\circ}\right]_{s}$ square plates for two different boundary conditions $\left(\alpha_{0}=10^{-6} 1 /{ }^{\circ} \mathrm{C}\right)$

\begin{tabular}{ccc}
\hline Boundary conditions & Present & Kant and Babu $^{7}$ \\
\hline SSSS & 0.0997 & 0.0997 \\
CCCC & 0.3354 & 0.3352 \\
\hline
\end{tabular}


Table 5 Effect of CNTs on the critical buckling temperatures of simply supported sandwich plates for various geometrical parameters $(a / h=50)$

\begin{tabular}{|c|c|c|c|c|c|c|}
\hline \multicolumn{2}{|c|}{ Geometrical Parameters } & \multicolumn{2}{|c|}{ Carbon/epoxy sheets } & \multicolumn{2}{|c|}{ Nanocomposite sheets } & \multirow{2}{*}{ Enhancement $\%$} \\
\hline$(a, b)$ & $h_{f} / h$ & $\Delta T_{c r}\left({ }^{\circ} \mathrm{C}\right)$ & $(m, n)$ & $\Delta T_{c r}\left({ }^{o} C\right)$ & $(m, n)$ & \\
\hline \multirow{3}{*}{$(0.5,0.25)$} & 0.05 & 97.4 & $(1,1)$ & 121.0 & $(1,1)$ & 24.2 \\
\hline & 0.10 & 54.4 & $(1,1)$ & 67.0 & $(1,1)$ & 23.2 \\
\hline & 0.15 & 39.3 & $(1,1)$ & 48.5 & $(1,1)$ & 23.4 \\
\hline \multirow[t]{3}{*}{$(0.5,0.5)$} & 0.05 & 82.7 & $(1,1)$ & 106.3 & $(1,1)$ & 28.5 \\
\hline & 0.10 & 50.4 & $(1,1)$ & 63.7 & $(1,1)$ & 26.4 \\
\hline & 0.15 & 37.7 & $(1,1)$ & 47.5 & $(1,1)$ & 26.0 \\
\hline \multirow[t]{3}{*}{$(0.5,0.75)$} & 0.05 & 82.6 & $(1,1)$ & 107.0 & $(1,1)$ & 29.5 \\
\hline & 0.10 & 50.9 & $(1,1)$ & 64.6 & $(1,1)$ & 26.9 \\
\hline & 0.15 & 38.0 & $(1,1)$ & 47.5 & $(1,2)$ & 25.0 \\
\hline
\end{tabular}

Table 6 Effect of CNTs on the critical buckling temperatures of simply supported sandwich plates for different stacking sequences of layers and values of $h_{f} / h$ parameter $(a=0.5 m, b=0.25 m, a / h=50)$

\begin{tabular}{|c|c|c|c|c|c|c|}
\hline \multirow{2}{*}{ Layup } & \multirow{2}{*}{$h_{f} / h$} & \multicolumn{2}{|c|}{ Carbon/epoxy sheets } & \multicolumn{2}{|c|}{ Nanocomposite sheets } & \multirow{2}{*}{ Enhancement \% } \\
\hline & & $\Delta T_{c r}\left({ }^{o} C\right)$ & $(m, n)$ & $\Delta T_{c r}\left({ }^{o} C\right)$ & $(m, n)$ & \\
\hline \multirow[t]{3}{*}[0^{\circ}/90^{\circ}/0^{\circ}]{} & 0.05 & 97.4 & $(1,1)$ & 121.0 & $(1,1)$ & 24.2 \\
\hline & 0.10 & 54.4 & $(1,1)$ & 67.0 & $(1,1)$ & 23.2 \\
\hline & 0.15 & 39.3 & $(1,1)$ & 48.5 & $(1,1)$ & 23.4 \\
\hline \multirow[t]{3}{*}[90^{\circ}/0^{\circ}/90^{\circ}]{} & 0.05 & 113.9 & $(3,1)$ & 139.3 & $(4,1)$ & 22.3 \\
\hline & 0.10 & 62.1 & $(4,1)$ & 76.1 & $(4,1)$ & 22.5 \\
\hline & 0.15 & 47.7 & $(3,1)$ & 59.4 & $(3,1)$ & 24.5 \\
\hline \multirow[t]{3}{*}[0^{\circ}/90^{\circ}]{$_{s}$} & 0.05 & 108.9 & $(1,1)$ & 135.7 & $(1,1)$ & 24.6 \\
\hline & 0.10 & 60.3 & $(1,1)$ & 74.6 & $(1,1)$ & 23.7 \\
\hline & 0.15 & 43.9 & $(1,1)$ & 54.6 & $(1,1)$ & 24.4 \\
\hline \multirow[t]{3}{*}[90^{\circ}/0^{\circ}]{$_{s}$} & 0.05 & 109.6 & $(1,1)$ & 136.8 & $(1,1)$ & 24.8 \\
\hline & 0.10 & 63.2 & $(1,1)$ & 78.9 & $(1,1)$ & 24.8 \\
\hline & 0.15 & 50.0 & $(2,1)$ & 62.9 & $(2,1)$ & 25.8 \\
\hline
\end{tabular}


Table 7 Effect of CNTs on the critical buckling temperature of sandwich plates for various types of boundary conditions and lay-ups $\left(a=b=0.5 m, a / h=50, h_{f} / h=0.05\right)$

\begin{tabular}{ccccc}
\hline \multirow{2}{*}{$\begin{array}{c}\text { Boundary } \\
\text { Conditions }\end{array}$} & Layup & \multicolumn{2}{c}{$\Delta T_{c r}\left({ }^{\circ} C\right)$} & Enhancement \% \\
\cline { 3 - 4 } SSSS & {$\left[0^{\circ} / 90^{\circ} / 0^{\circ}\right]$} & $\begin{array}{c}\text { Carbon/epoxy } \\
\text { sheets }\end{array}$ & $\begin{array}{c}\text { Nanocomposite } \\
\text { sheets }\end{array}$ & \\
& {$\left[0^{\circ} / 90^{\circ} / 0^{\circ} / 0^{\circ}\right]$} & 82.7 & 106.3 & 28.5 \\
& {$\left[0^{\circ} / 90^{\prime} / 0^{\circ} / 90^{\circ} / 0^{\circ}\right]$} & 84.1 & 108.0 & 28.4 \\
CSCS & {$\left[0^{\circ} / 90^{\circ} / 0^{\circ}\right]$} & 83.6 & 107.4 & 28.5 \\
& {$\left[0^{\circ} / 90^{\circ} / 90^{\circ} / 0^{\circ}\right]$} & & & \\
& {$\left[0^{\circ} / 90^{\prime} / 0^{\circ} / 90^{\circ} / 0^{\circ}\right]$} & 96.1 & 120.7 & 25.6 \\
& & 99.2 & 125.0 & 26.0 \\
CCCC & {$\left[0^{\circ} / 90^{\circ} / 0^{\circ}\right]$} & 97.6 & 122.9 & 25.9 \\
& {$\left[0^{\circ} / 90^{\circ} / 90^{\circ} / 0^{\circ}\right]$} & 105.1 & & 23.7 \\
& {$\left[0^{\circ} / 90^{\circ} / 0^{\circ} / 90^{\circ} / 0^{\circ}\right]$} & 113.7 & 130.0 & 24.2 \\
\hline
\end{tabular}

Table 8 Effect of CNTs on the critical buckling temperature of sandwich plates for various types of boundary conditions and different values of $b / a$ parameter $\left(a=0.5 m, a / h=50, h_{f} / h=0.05\right)$

\begin{tabular}{|c|c|c|c|c|}
\hline \multirow{2}{*}{$\begin{array}{l}\text { Boundary } \\
\text { Conditions }\end{array}$} & \multirow{2}{*}{$\mathrm{b} / \mathrm{a}$} & \multicolumn{2}{|c|}{$\Delta T_{c r}\left({ }^{o} C\right)$} & \multirow{2}{*}{ Enhancement $\%$} \\
\hline & & Carbon/epoxy sheets & Nanocomposite sheets & \\
\hline \multirow[t]{3}{*}{ SSSS } & 0.5 & 97.4 & 121.0 & 24.2 \\
\hline & 1 & 82.7 & 106.3 & 28.5 \\
\hline & 1.5 & 82.6 & 107.0 & 29.5 \\
\hline \multirow[t]{3}{*}{ CSCS } & 0.5 & 101.3 & 125.3 & 23.7 \\
\hline & 1 & 96.1 & 120.7 & 25.6 \\
\hline & 1.5 & 96.2 & 120.5 & 25.3 \\
\hline \multirow[t]{3}{*}{$\mathrm{CCCC}$} & 0.5 & 110.1 & 134.6 & 22.3 \\
\hline & 1 & 105.1 & 130.0 & 23.7 \\
\hline & 1.5 & 101.8 & 126.0 & 23.8 \\
\hline
\end{tabular}

